Anales de Geografía de la Universidad Complutense ISSN: 0211-9803

http://dx.doi.org/10.5209/AGUC.69338

\title{
Análisis de la calidad visual del paisaje del Parque Nacional de la Sierra de Guadarrama mediante procedimientos indirectos: EMC y SIG
}

\author{
Alejandro Vallina Rodríguez ${ }^{1}$ \\ Recibido: 18 de septiembre del 2017 / Enviado a evaluar: 16 de noviembre del 2017/ Aceptado: 9 de diciembre del 2019
}

Resumen. Frente a la complejidad que supone la valoración objetiva del paisaje, la presente investigación propone una aproximación metodológica desde la evaluación indirecta de uno de los tres grandes bloques (intrínsecos, de incidencia positiva y de incidencia negativa) que forman la calidad visual de cualquier espacio, atendiendo fundamentalmente a los valores intrínsecos. El objetivo principal del análisis consistirá en descomponer todos aquellos valores y criterios que forman parte de los elementos visuales inherentes del paisaje de la Sierra de Guadarrama para, a partir de la utilización de las metodologías de evaluación multicriterio (EMC) y su combinación con las posibilidades que ofrecen los sistemas de Información Geográfica (SIG), llegar a un conocimiento suficiente de la realidad visual del entorno. Esto permitirá interpretar técnicamente los diversos factores que configuran los valores intrínsecos del Guadarrama, integrando los resultados en una cartografía temática del ámbito, que muestre detalladamente los valores de calidad visual de forma territorializada.

Palabras clave: Calidad visual; evaluación del paisaje; EMC; SIG.

\section{[en] Analysis of the visual quality of the landscape of the Sierra de Guadarrama National Park using indirect procedures: EMC and GIS}

\begin{abstract}
Faced with the complexity involved in the objective assessment of the landscape, the present investigation proposes a methodological approach from the indirect evaluation of one of the three large blocks (intrinsic, positive and negative incidence) that form the visual quality of any space, fundamentally attending to intrinsic values. The main objective of the analysis will be to decompose all those values and criteria that are part of the inherent visual elements of the landscape of the Sierra de Guadarrama to, based on the use of multicriteria evaluation methodologies (MCE) and their combination with the possibilities offered by Geographic Information Systems (GIS), to get enough knowledge of the visual reality of the environment This will allow to interpret technically the diverse factors that configure the intrinsic values of the Guadarrama, integrating the results in a thematic cartography of the field, which shows in detail the values of visual quality in a territorialized way.
\end{abstract}

Keywords: Visual quality; landscape assessment; MCE; GIS.

1 Departamento de Geografía. Universidad Autónoma de Madrid.

E-mail: alejandro.vallina@predoc.uam.es 


\section{[fr] Analyse de la qualité visuelle du paysage du parc national de la Sierra de Guadarrama à l'aide de procédures indirectes: EMC et SIG}

Résumé. Face à la complexité de l'évaluation objective du paysage, la présente enquête propose une approche méthodologique à partir de l'évaluation indirecte de l'un des trois grands blocs (incidence intrinsèque, positive et négative) qui forment la qualité visuelle de tout espace, assister fondamentalement aux valeurs intrinsèques. L'objectif principal de l'analyse sera de décomposer toutes les valeurs et tous les critères qui font partie des éléments visuels inhérents au paysage de la Sierra de Guadarrama à, de l'utilisation de méthodologies d'évaluation multicritères (MEC) et leur combinaison avec les possibilités offerts par les Systèmes d'Information Géographique (SIG), pour atteindre une connaissance suffisante de la réalité visuelle de l'environnement. Cela permettra d'interpréter techniquement les divers facteurs qui configurent les valeurs intrinsèques du Guadarrama, en intégrant les résultats dans une cartographie thématique du champ, qui montre en détail les valeurs de la qualité visuelle d'une manière territorialisée.

Mots clés: Qualité visuelle; évaluation du paysage; EMC, SIG.

Cómo citar. Vallina Rodríguez, A. (2020): Análisis de la calidad visual del paisaje del Parque Nacional de la Sierra de Guadarrama mediante procedimientos indirectos: EMC y SIG. Anales de Geografía de la Universidad Complutense, 40(1), 183-215.

Sumario. 1. Introducción. 2. Objetivos y área de estudio. 3. Fuentes. 4. Metodología. 4.1 Determinación de los componentes. 4.2 Normalización y valoración. 4.2.1 Fisiografía. 4.2.2. Vegetación. 4.2.3. Usos del suelo. 4.2.4. Elementos singulares. 4.3. Ponderación de los criterios y aplicación del algoritmo EMC. 4.3.1. Superposiciones ponderadas de los criterios de evaluación. 5. Resultados y conclusiones. 6. Referencias bibliográficas.

\section{Introducción}

Tanto para la comunidad científica, como para la sociedad en su conjunto, la asunción de las directrices contenidas en el Convenio Europeo del Paisaje (2000) supuso una revisión metodológica sobre la relación que guardan los componentes del espacio observables de una manera asequible por el sujeto medio y la composición espaciotemporal que presentan. De este modo, el acercamiento al paisaje desde la perspectiva científica pasa a incorporar signos particulares, a través de los cuales hacen llegar su identidad al observador (Saeidi et al., 2017), al mismo tiempo que le transmiten evocaciones de naturaleza estética. El paisaje ya no es visto y tratado como una combinación de ciencias, si no que se sitúa por encima de éstas para trocar en una forma universal de observar el medio. Es por ello que, desde este trabajo, el paisaje ha adoptado la concepción de paisaje realizada por Cancer (1994), que lo define como un conjunto de hechos visibles o invisibles, de los que el observador percibe, en un momento dado, un resultado global de un territorio.

De este modo, si el paisaje posee una evidente exteriorización de carácter visual, parece coherente considerar en su análisis también al propio observador y a las evocaciones o sensaciones de naturaleza estética que éste percibe. A este plano de análisis las directrices y determinaciones del CEP añadieron la necesidad de tratamiento de igual a igual entre el paisaje y los demás recursos del territorio. Frente 
a estos nuevos desafíos que el campo del conocimiento sobre el paisaje plantea, las ciencias, y más concretamente la disciplina geográfica, reaccionó hace décadas con el surgimiento (Hermes et al., 2018) de nuevas técnicas y procedimientos de valoración y objetivación estética y/o ambiental acerca del paisaje percibido, esto es, el paisaje visual.

Las diferentes metodologías de valoración de la calidad visual del paisaje son la respuesta que las dinámicas del conocimiento han producido a lo largo de las últimas décadas, con la vista puesta en el establecimiento de respuestas a la hora de gestionar las preferencias de la ciudadanía. Las determinaciones que desde cualquier disciplina científica puedan hacerse sobre la calidad visual se encuentran entre los aspectos más difíciles de cuantificar en lo tocante al medio ambiente, ya que en sus cálculos se han de equilibrar constantemente la naturalidad y la conservación de los valores preeminentes (Franch- Pardo y Cáncer, 2017). Todas estas estimaciones, claro está, se realizan en un plano teórico, pero tienen un reflejo empírico basado en paisajes concretos, con múltiples y variados intereses para la población, y donde habrá de tenerse en cuenta los aprovechamientos que se desarrollen o puedan desarrollarse en los mismos. Dos son los componentes a los que se recurrirá generalmente en la determinación del valor global de calidad paisajística: la calidad y la fragilidad visual, pudiendo ser ambas evaluadas mediante metodologías directas o indirectas. La presente investigación únicamente considerará la calidad visual como fundamento de la evaluación paisajística, entendida como el grado de excelencia de éste, su mérito para que su esencia, su estructura actual se conserve (Ramos y González, 1987). Dentro de la calidad visual, componente que en el campo de los estudios del paisaje ha solido dividir en tres indicadores: calidad intrínseca, valores de incidencia paisajística positiva y valores de incidencia paisajística negativa, interesará en esta investigación la aproximación a los denominados valores intrínsecos del paisaje, es decir, los valores esenciales del paisaje guadarrameño sin los cuáles éste no podría identificarse, ya que le dan sentido y le aportan el significado actual.

Debido a la naturaleza y multiplicidad de los datos y los análisis necesarios, el abordaje metodológico de la presente investigación se realizó empleando la Evaluación Multicriterio (EMC) con apoyo en Sistemas de Información Geográfica (SIG), en concreto, el software ArcGIS 10.4. Las alternativas adoptadas en el proceso de toma de decisiones se configuran como las más idóneas para el decisor y el objetivo, cuestión no exenta de un grado mayor o menor de subjetividad, y que entra a formar parte del resultado y será ponderada de forma ecuánime en la investigación. De forma más concreta, para paliar los posibles conflictos de subjetividad, se utilizarán técnicas EMC, y más concretamente el uso del denominado Modelo analítico Jerárquico (AHP), métodos de ponderación aceptados internacionalmente y que tienen clara aplicación en el estudio del paisaje. En la presente investigación, estas técnicas han sido de utilidad para descomponer las relaciones complejas de los componentes intrínsecos del paisaje, ordenándolos en una estructura jerárquica donde se obtienen valores numéricos válidos para la valoración de la calidad visual (Karanik et al., 2016). Por otra parte, la integración del análisis multicriterio en los SIG permite combinar y valorar simultáneamente los criterios (las bases para la toma de 
decisión) con sus factores (los aspectos que los hacen fuertes o los debilitan) a través del uso de sus atributos (las variables) dentro de unas determinadas reglas de decisión y valoración.

\section{Objetivos y área de estudio}

El objetivo fundamental de la investigación que aquí se presenta pasa por la consideración del paisaje desde el punto de vista exclusivamente visual, centrándose en los aspectos derivados de la percepción para concretar una metodología de identificación y valoración de los elementos inmanentes y característicos, esto es, los valores intrínsecos, de una de las zonas más emblemáticas de la geografía del interior de la meseta Ibérica. Dicha búsqueda de elementos del paisaje se ha propuesto desde la descomposición de éste, según sus elementos visuales principales. Con la vista puesta en la generación de un método independiente, en el que la valoración de la calidad visual del paisaje se realiza de forma indirecta por expertos, que descomponen en atributos cada valor visible e invisible del territorio, se ha buscado que la subjetividad quede implícita y pueda controlarse mediante un criterio uniforme, y la evaluación emane de la contemplación del paisaje. La disgregación del paisaje en componentes persigue la elaboración de una imagen completa de los valores (de la Fuente de Val et al., 2004) contenidos en el paisaje propio del Parque Nacional de la Sierra de Guadarrama. Además de los propios confines del Parque Nacional de la Sierra de Guadarrama, se ha estudiado su área de influencia, no sólo desde el punto de vista visual, también económico, social, territorial, contemplado en la Figura 1 como Área de influencia visual. Esta selección de componentes o factores visuales del paisaje será la base de la generación del método que aquí se propone. Para ello, es necesario comprender que el área de trabajo, El Parque Nacional de la Sierra de Guadarrama (Comunidad Autónoma de Madrid, España) y su zona de influencia, se ha seleccionado por la diversidad y las relaciones, a menudo complejas, de sus valores naturales y socioculturales.

El marco seleccionado en la investigación es parte de la división natural entre las mesetas norte y sur en la zona centro de la Península Ibérica, convirtiéndose así en paso casi obligado entre las dos Castillas. Situada entre las sierras de Gredos y de Ayllón, este cordal se extiende como una sucesión de muros continuos de roquedos viejos en dirección suroeste-noreste por las provincias de Madrid, Segovia y Ávila, creando así un paisaje de enorme valor debido a la unión de sus altas cumbres, sus aristas torreadas, sus circos y lagunas de origen glaciar, los amplios valles y laderas que los dominan y sus extensas arboledas y gargantas (Rivera et al, 2014). El Guadarrama se considera, desde esta investigación, un paisaje de elevada complejidad, ya que de forma recurrente en su territorio se hacen patentes las relaciones entre los excelsos valores naturales que alberga y la acción humana, que se muestra en aspectos tan fundamentales como la presión de usos del suelo, la generación de infraestructuras o los impactos por actividades turísticas, entre otros. 
Figura 1. Cartografía de situación.

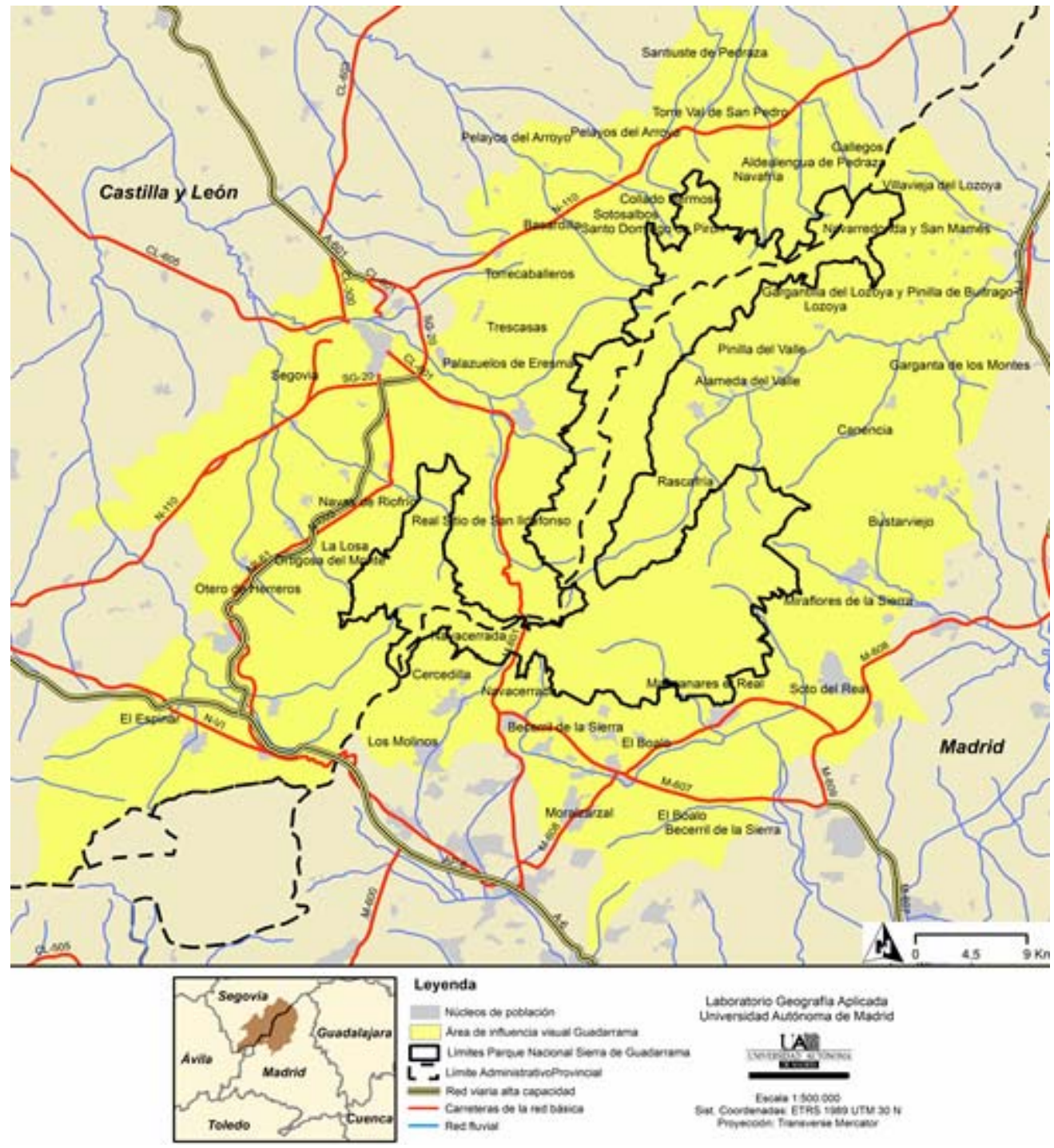

Fuente: Elaboración propia a partir de bases cartográficas del IGN, 2018

No se debe olvidar que el área del Parque Nacional de la Sierra de Guadarrama se encuentra a una distancia relativamente corta con respecto al área urbana y metropolitana de Madrid, con la presión antrópico-demográfica que ello genera. A pesar de lo variado de sus elementos, la Sierra de Guadarrama puede considerarse como una unidad homogénea desde el punto de vista orográfico, un cuerpo simple 
que se ha modelado a lo largo de milenios con una faceta natural y otra marcadamente humana, lo que ha imprimido en su fisonomía un fondo cultural perfectamente identificable a lo largo y ancho de sus valles, ríos y montañas. Su condición de frontera natural y estratégica ha hecho que, desde tiempos inmemoriales, sus habitantes trazaran distintas rutas para controlarla y franquearla.

\section{Fuentes}

La presente investigación está fundamentada en el análisis perceptivo de los elementos del paisaje, para cuyo ajuste se ha procedido a trabajar previamente con información geolocalizada de tipo digital. En la conformación del modelo de decisión EMC han tenido una importancia capital las capas de criterios señaladas en la Tabla 1, donde se aporta información relativa tanto a sus características esenciales, el modelo de datos trabajado y la escala a la que se encuentra referenciada la información. Las capas, de libre acceso en las Infraestructuras de Datos Espaciales (IDEs), han sido descargadas del Instituto Geográfico Nacional (IGN) y del Ministerio para la Transición Ecológica (MITECO). La normalización de cada una de la información contenida en las capas referidas se ha elaborado de acuerdo con la referencia de la máscara de límites municipales, de resolución 1:25.000 y proyección en sistema geodésico UTM30N-ETRS89 (Tabla 1).

Tabla 1. Fuentes de datos.

\begin{tabular}{|c|c|c|c|c|}
\hline ELEMENTO & AUTOR & FORMATO & $\begin{array}{l}\text { ESCALA/ } \\
\text { RESOL. }\end{array}$ & Proyección \\
\hline Límites municipales & IGN & Vectorial/SHP & $1: 25000$ & UTM30N-ETRS89 \\
\hline Base cartográfica & $\begin{array}{c}\text { IGN/ } \\
\text { BTN100 }\end{array}$ & Vectorial/SHP & $1: 100000$ & UTM29N-ETRS89 \\
\hline $\begin{array}{l}\text { Modelo digital eleva- } \\
\text { ción }\end{array}$ & $\begin{array}{c}\text { IGN/ } \\
\text { MTN25 }\end{array}$ & $\begin{array}{l}\text { Ráster/ } \\
\text { ASCI }\end{array}$ & $25 \mathrm{~m}$ & UTM30N-ETRS89 \\
\hline $\begin{array}{l}\text { Base ocupación del } \\
\text { suelo }\end{array}$ & IGN/ CLC11 & $\begin{array}{l}\text { Ráster/ } \\
\text { MMZ }\end{array}$ & $1: 100000$ & UTM30N-ETRS89 \\
\hline Mapa forestal español & $\begin{array}{l}\text { MITECO } \\
\text { MFE50 }\end{array}$ & $\begin{array}{l}\text { Ráster/ } \\
\text { SHP }\end{array}$ & $1: 50000$ & UTM30-ETRS89 \\
\hline $\begin{array}{c}\text { Espacios naturales } \\
\text { protegidos }\end{array}$ & MITECO & Vectorial/SHP & $1: 25000$ & UTM30-ETRS89 \\
\hline
\end{tabular}

Fuente: Elaboración propia según metadatos del MITECO e IGN 


\section{Metodología}

La Calidad Visual de los elementos intrínsecos del paisaje del Parque Nacional de la Sierra de Guadarrama y el ámbito territorial de su entorno más inmediato se determinará, tal y como ya se ha comentado con anterioridad, según una valoración directa del medio. En el proceso de valoración, que parte de la identificación de los elementos constituyentes del medio, se ha utilizado la metodología de evaluación multicriterio (EMC) para adaptar las decisiones procedimentales a las características del ámbito de estudio, otorgando para ello relevancias relativas para los criterios paisajísticos contemplados.

\subsection{Determinación de los componentes}

El estudio de la calidad visual del paisaje se fundamenta en una valoración del medio en un momento concreto, partiendo de la selección de los elementos o componentes que lo integran (Vallina, 2017). El modelo de valoración, capaz de organizar con detalle los componentes que integran la realidad paisajística del entorno, se ha elaborado en torno a un gran grupo general de criterios, los elementos de calidad intrínseca, dentro de los cuales encontramos sobre todo factores de origen natural, aunque existen también de origen antrópico. Entre dichos valores se han destacado principalmente los rasgos geomorfológicos, de cubiertas vegetales y usos de suelo y se ha valorado positivamente la existencia de elementos considerados como singulares. El modelo de calidad intrínseca del paisaje incluye los siguientes factores y criterios de valoración (Gráfico 1).

Gráfico 1. Criterios de evaluación de la calidad intrínseca del paisaje

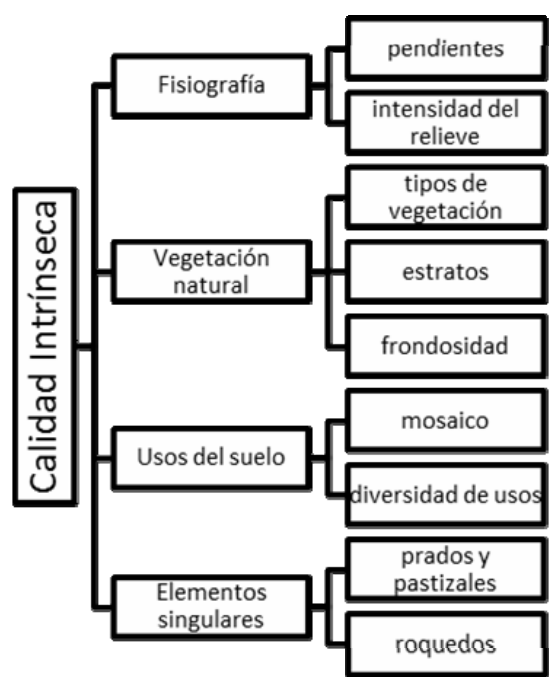

Fuente: Elaboración propia (Vallina, 2017) 


\subsection{Normalización y valoración}

Una vez definido el modelo de componentes a considerar en el estudio de paisaje deben establecerse unas pautas de valoración de cada valor integrante del modelo. La valoración se efectúa en una escala jerárquica similar para todos los componentes, de manera que cada uno de ellos es tratado de forma independiente.

\subsubsection{Fisiografía}

La fisiografía como elemento que le confiere un valor distintivo al paisaje ha sido empleada como criterio en numerosos estudios de paisaje (Alberruche et al, 2015). Cuando se están cotejando las propiedades visuales de paisajes complejos desde el punto de vista geomorfológico, como ocurre con la Sierra de Guadarrama, las geoformas sobresalientes deben tener al menos 25 metros de desnivel respecto los puntos adyacentes para que sea posible distinguirlas del entorno y, en consecuencia, infieran carácter al paisaje (Tress, Tress, Decamps, y D`Hausteserre, 2001). Teniendo en cuenta esta consideración el trabajo de investigación ha seleccionado como base del tratamiento de información digital del terreno una ventana móvil de $3 \times 3$ píxeles, que ocupa en la realidad aproximadamente $73 \mathrm{ha}$, por entender que ésta es la superficie múltiple del tamaño de píxel mínimamente extensa como para percibir estas unidades de un modo significativo a nivel de calidad visual del paisaje.

El mapa de fisiografía se ha elaborado, por lo tanto, a partir del mapa de pendientes y del mapa de intensidad del relieve. La pendiente se calculó a partir del Modelo Digital de Elevación MDE (Figura 2) empleando la herramienta de análisis espacial para cálculo de pendientes que incorpora el hardware ArcGIS.

Figura 2. Modelo digital de elevaciones área del Guadarrama

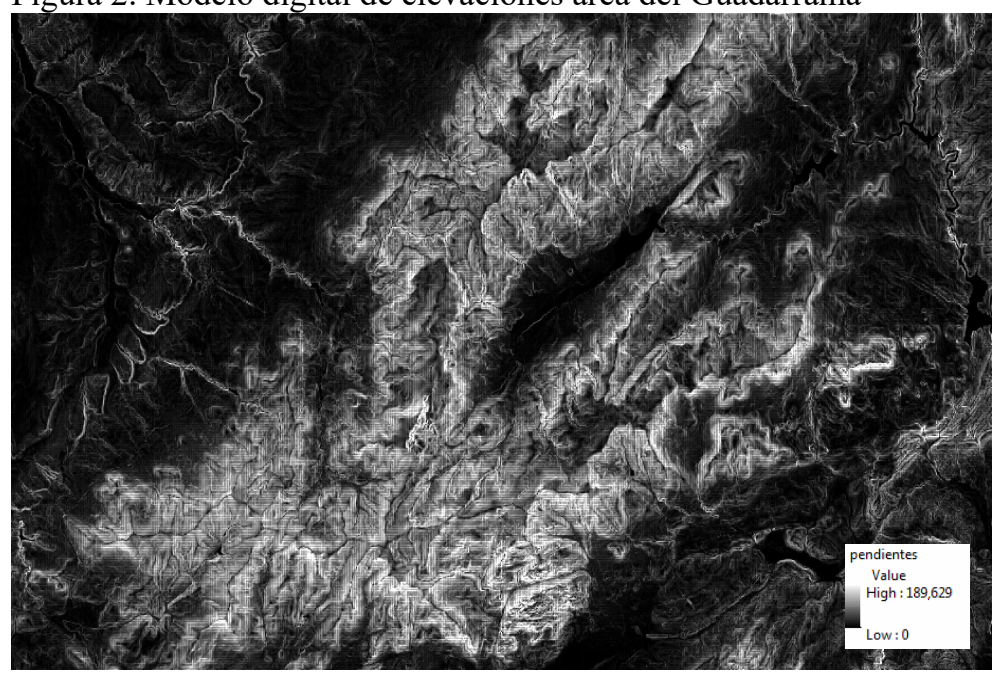

Fuente: Elaboración a partir de modelo de datos IGN 1:25000 
Tabla 2. Valores de reclasificación y normalización de las pendientes de la superficie

\begin{tabular}{|c|c|c|}
\hline Criterios valoración & Valores de pendiente & Valores de normalización \\
\hline Relieves llanos y suaves & $0-15 \%$ & 6 \\
\hline Pendientes moderadas & $15-30 \%$ & 7 \\
\hline Fuertes pendientes & $30-60 \%$ & 8 \\
\hline Relieves escarpados & $>60 \%$ & 10 \\
\hline
\end{tabular}

Fuente: Elaboración propia según el criterio de normalización de Alberruche et al (2015)

Una vez obtenida la capa de pendientes, y a partir del MDE, se procedió simplemente a reclasificar dicha capa (Tabla 2), según un cierto número de intervalos de pendiente (Alberruche et al., 2015) que permiten caracterizarla con un detalle apropiado a la escala de trabajo, y se ajustaron los valores a la realidad fisiográfica del Guadarrama para así asignarle un valor de calidad visual adecuado a los paisajes existentes (Figura 3).

Figura 3. Asignación de valores de calidad visual según el criterio pendiente

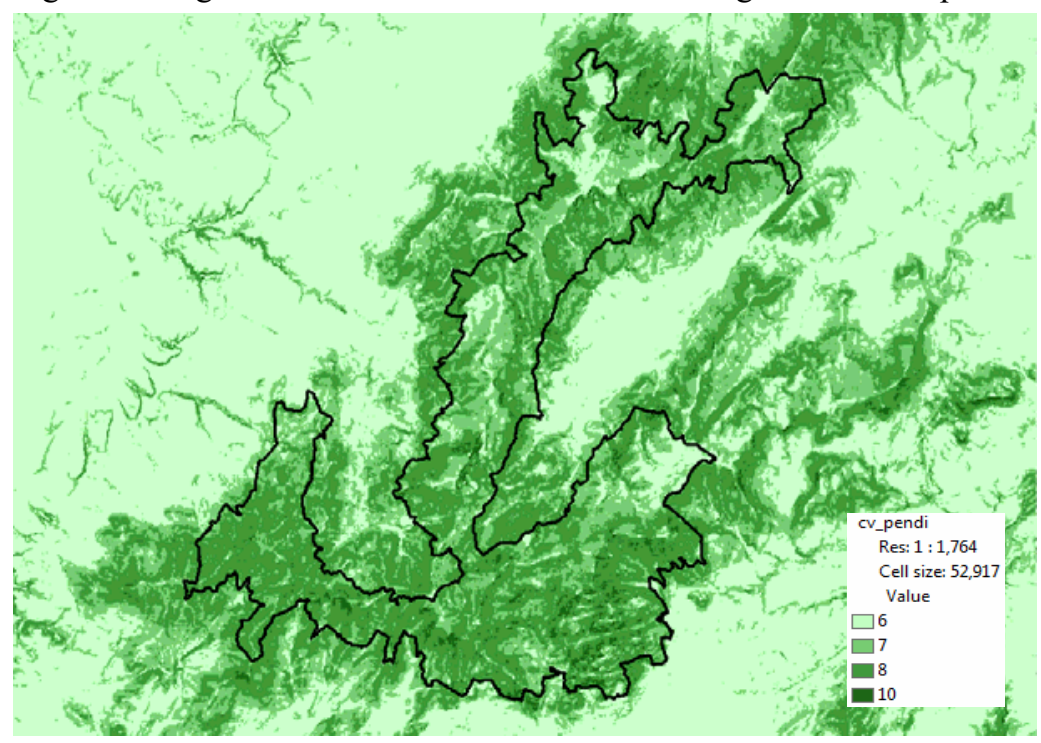

Fuente: Elaboración propia según modelo AHP

Por su parte la valoración de la intensidad del relieve propuesta busca un método de evaluación de la morfología del relieve de forma indirecta, a través el desnivel observado en un ámbito específico del territorio. Para la asignación de los valores de la calidad visual se consideró que un desnivel mayor o igual a $20 \mathrm{~m}$ permite percibir un cambio en la intensidad del relieve.

Este parámetro, por tanto, se ha evaluado usando estadística de bloques, en bloques regulares de 9 unidades ( 3 x 3 celdas), para obtener 2 capas: una con los valores de altitud máximos (opción MAXIMUM) y otra con los mínimos 
(MINIMUM). Con la obtención de estas capas y su posterior resta se averiguó la diferencia entre el punto más alto y el más bajo entre los bloques de celdas seleccionados (Figura 4). Sobre la capa de desniveles que se ha construido se realiza una reclasificación mediante 5 valores de normalización (Tabla 3), de acuerdo con los estándares de clasificación de la calidad visual.

Figura 4. Estadística de bloques de desnivel acumulado.

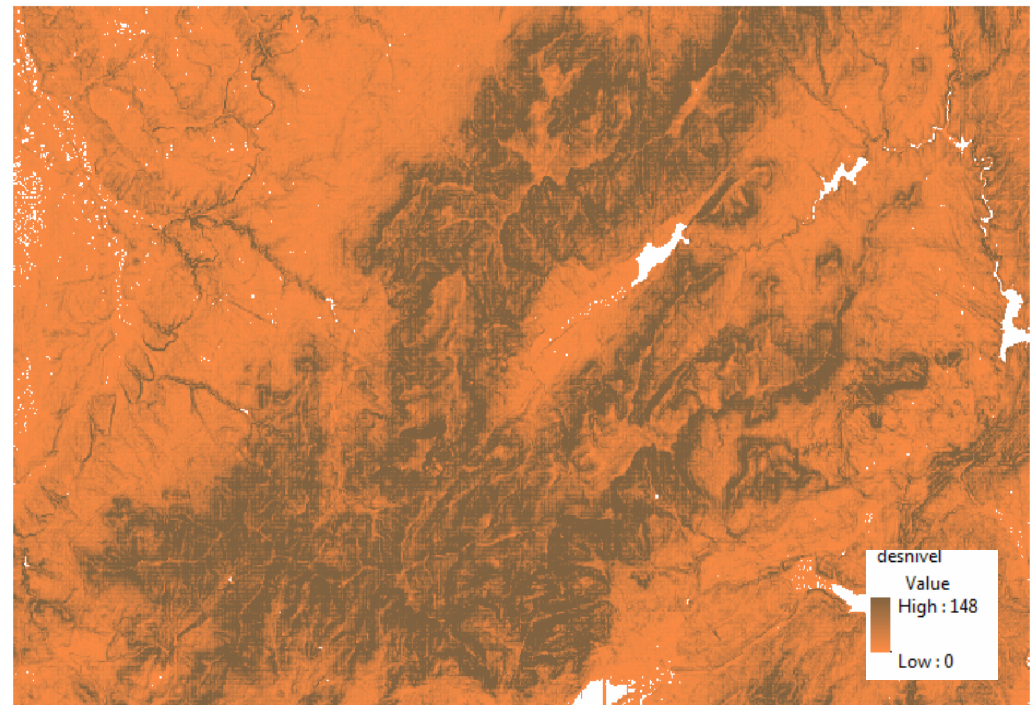

Fuente: Elaboración propia.

Tabla 3. Valores de normalización de desniveles

\begin{tabular}{|c|c|}
\hline Desnivel promedio del bloque (3x3 celdas) & Valores de normalización \\
\hline $0-25 \mathrm{~m}$ & 2 \\
\hline $26-40 \mathrm{~m}$ & 4 \\
\hline $41-60 \mathrm{~m}$ & 6 \\
\hline $61-80 \mathrm{~m}$ & 8 \\
\hline $80-148 \mathrm{~m}$ & 10 \\
\hline
\end{tabular}

Fuente: Elaboración propia según el criterio de normalización de Alberruche et al (2015)

Posteriormente, y usando estas dos capas de información altimétrica, se calculó la diferencia entre ellas, por medio del álgebra de mapas (calculadora Raster), para obtener los desniveles existentes en metros. Finalmente, según los valores de desnivel general obtenidos mediante la sustracción de las capas, se establecieron clases de intensidad del relieve (Figura 5). 
Figura 5. Calidad visual de la intensidad del relieve. Sierra de Guadarrama.

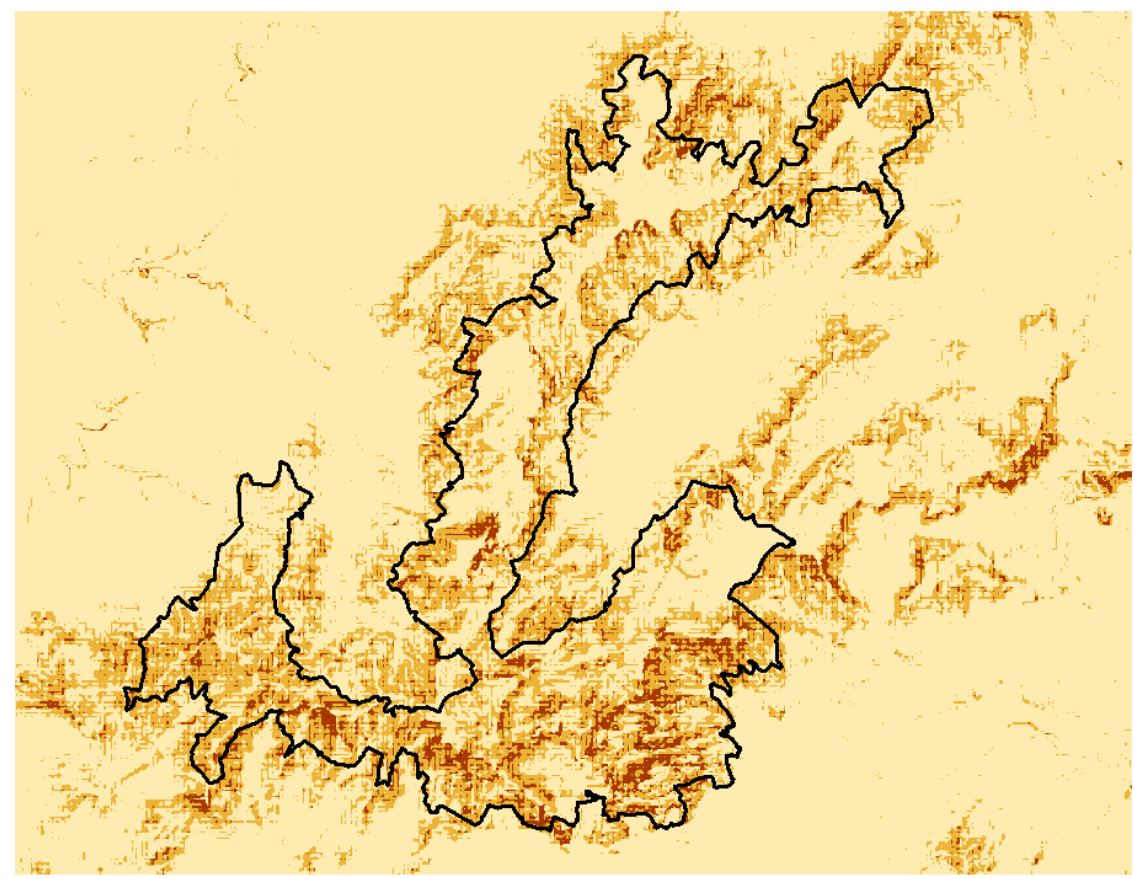

Fuente: Elaboración propia según modelo AHP

\subsubsection{Vegetación}

Como manto que recubre la superficie de la tierra la incidencia de la vegetación en la percepción visual del paisaje de un determinado entorno es innegable. Por ello, con el objetivo de focalizar el esfuerzo investigador hacia la sistematización de un procedimiento que genere consenso, este trabajo ha adoptado como suya la idea de que la descripción visual del componente vegetación debe incluir dos perspectivas de análisis básicas, el estudio florístico y el fisonómico.

En esta investigación se han adaptado las consideraciones realizadas por Panareda (2009), de tal modo que el acercamiento a las características florísticas de la vegetación de la Sierra de Guadarrama se hará desde la inclusión del examen de los tipos de especies presentes y las comunidades vegetales relevantes desde el punto de vista paisajístico-visual (Figura 6). Del mismo modo, el análisis de las características contemplará la percepción que el observador pueda tener sobre la estructura de las masas vegetales de la zona, así como los grados de cobertura observables, la estratificación y las características del follaje, esto último analizado desde el punto de vista de la frondosidad, que en posteriores líneas se analizará pormenorizadamente. 
Figura 6. Usos del suelo aplicados a la vegetación.
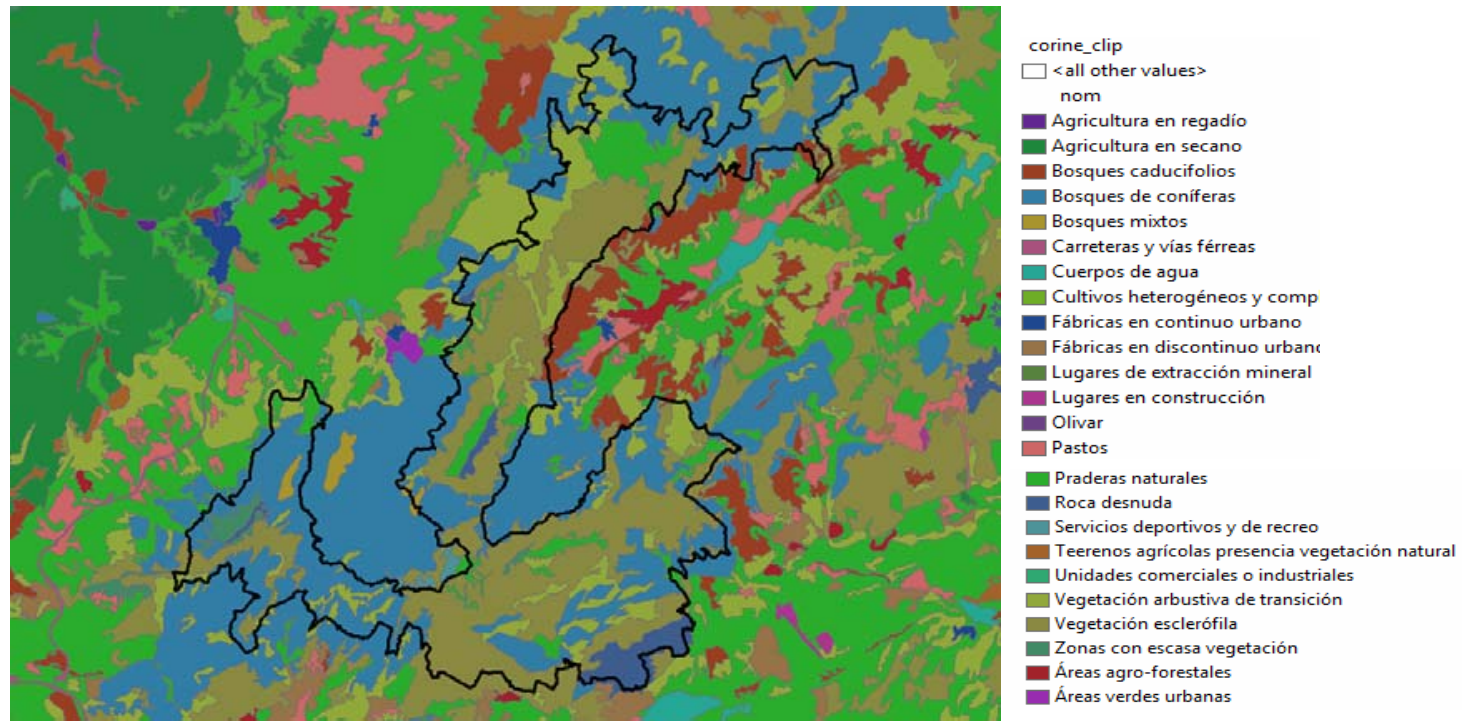

Fuente: Elaboración propia a través de datos del proyecto CLC 2011

Una vez concretadas las variables de evaluación de la vegetación, que caracterizan y tienen incidencia en la calidad paisajística de un lugar, esto es, los tipos de vegetación, estratos y frondosidad, se comenzó a operar con la capa de coberturas y usos del suelo Corine Land Cover 11 (CLC11), que contiene toda la información relevante sobre los tipos de vegetación que contiene cada malla del territorio. Para poder operar con la capa de usos del suelo lo primero que debe realizarse es una extracción de la información para la zona de estudio en concreto.

Una vez que se construyó la capa de Corine Land Cover para el ámbito territorial del Parque Nacional de la Sierra de Guadarrama y el ámbito paisajístico adyacente se trabajó con la base de datos asociada, concretamente con la vinculada a los tipos de vegetación. Ello quiere decir que de la capa de CLC11 se extrajeron todas aquellas informaciones territoriales que no estuvieran en relación con la vegetación, tales como espacios agrícolas, espacios urbanos, redes de transporte, etc. Para ello se trabaja en la tabla de atributos de la capa, añadiendo un campo de normalización de la calidad visual de los diferentes tipos de vegetación, basándose para ello en las investigaciones de Aramburu et al, (2005) (Tabla 4). 
Tabla 4. Valores de normalización de la calidad visual según tipos de vegetación

\begin{tabular}{|c|c|}
\hline Tipo de vegetación & Valor de Calidad Visual \\
\hline Agrícola con vegetación natural & 5 \\
\hline Bosques de coníferas & 10 \\
\hline Bosques de frondosas & 9 \\
\hline Bosques mixtos & 9 \\
\hline Espacios con escasa vegetación & 3 \\
\hline Matorral boscosos de transición & 6 \\
\hline Matorral esclerófilo & 6 \\
\hline Pastizales supraforestales naturales & 10 \\
\hline Prados y praderas & 7 \\
\hline Sistemas agroforestales adehesados & 8 \\
\hline No data (sin datos) & No data \\
\hline
\end{tabular}

Fuente: Elaboración propia según el criterio de normalización de Aramburu et al (2005)

Por aclarar más sobre las razones de la normalización propuesta en materia de tipologías de vegetación, es necesario indicar que la presente investigación ha considerado las propuestas generalistas de interpretación visual de diversos tipos de vegetación, señaladas en investigaciones como la de Aramburu et al. (2005) o Cohen et al. (2016). La presente investigación ha realizado su propuesta de normalización para los tipos de vegetación conociendo, primeramente, las especies arbustivas y vegetales existentes en el área de estudio.

A pesar de su aparente homogeneidad orográfica del área, ésta presenta un grado de diversidad significativo, en el que el relieve, abrupto y compartimentado en buena parte del entorno, determina la existencia de numerosos y muy marcados microclimas que, a su vez, permiten la aparición de facies "secas" y de facies húmedas o de montaña en la vegetación. El Parque Nacional de la Sierra de Guadarrama está situado en el domino de la montaña mediterránea, dominada por la vegetación esclerófila, muy bien adaptada a la sequía estival, que en sus versiones arbóreas han sido catalogadas como de alto valor paisajístico y en su forma arbustiva como de calidad media. En la montaña del Guadarrama, al igual que en otras de la Península Ibérica, las posiciones meridionales son mucho más secas, ya que no logran hacer desaparecer la aridez estival. Por esta razón el piso planocaducifolio no aparece y del bosque esclerófilo se pasa directamente al de coníferas, con diversas especies de pinos, que han sido catalogados con los valores más altos de calidad visual en el área de estudio, habida cuenta que estos poblamientos de coníferas constituyen, muchas veces, enclaves botánicos de gran valor que albergan los últimos cientos de individuos de algunas especies endémicas.

A partir de este proceso de normalización de valores se procede a realizar una reclasificación de la cartografía, de acuerdo con valores de calidad visual apreciables para cada uno de los tipos de vegetación existentes. 
Figura 7. Calidad visual según tipo de vegetación

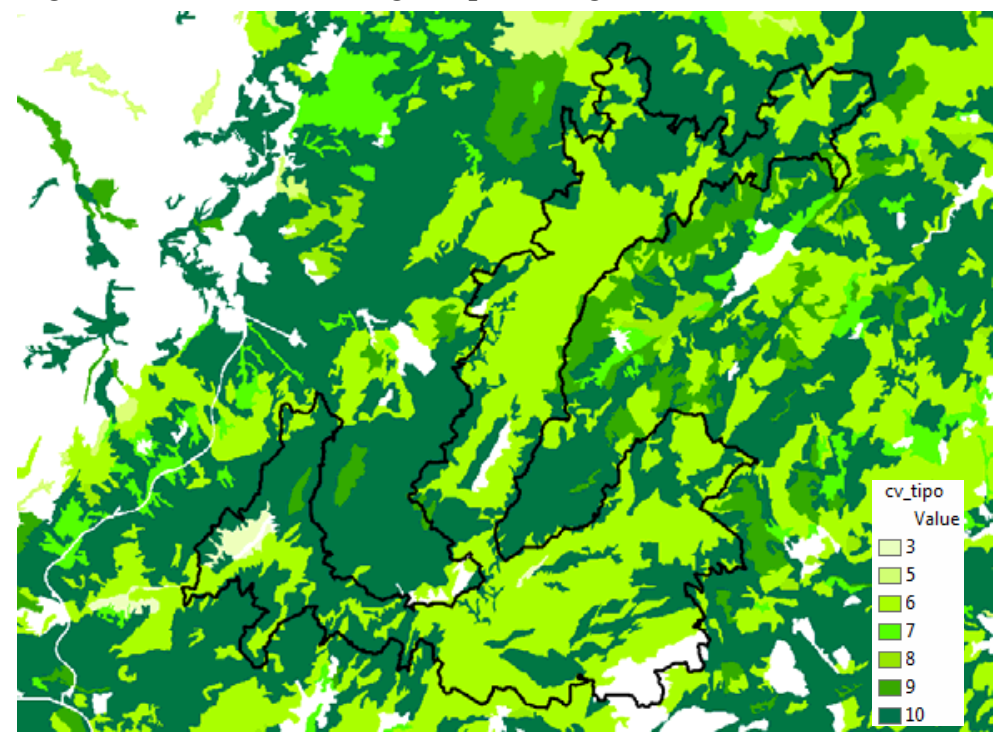

Fuente: Elaboración propia según modelo AHP

A partir de la base de datos ya trabajada se procede a realizar una extracción relativa a la información de los estratos de vegetación de la zona de estudio, añadiendo posteriormente, al igual que anteriormente, un campo de normalización de valores de la calidad visual (Alberruche, y otros, 2015) (Figura 7). La información normalizada se añadió a la base de datos dentro del SIG, con el objetivo de poder expresarla en una cartografía capaz de mostrar la calidad visual del paisaje de la Sierra de Guadarrama según los estratos de la vegetación existente (Tabla 5). El segundo de los aspectos fisionómicos de la vegetación que se ha tenido en cuenta en esta investigación, referido a su frondosidad, implica primeramente un desarrollo metodológico que determine de qué forma se puede calcular este índice. Algunos autores, entre los que destaca Serrada (2008), han tratado en profundidad el asunto de la frondosidad de la vegetación como el resultado intrínseco de la acción de la orientación de las superficies analizadas con respecto al sol. La frondosidad de la vegetación depende entre muchos factores de la disponibilidad de luz y agua. Es bien sabido que, por lo general, la orientación de las vertientes se asocia a variaciones climáticas debido a la mayor o menor exposición a factores externos, como los vientos, insolación o la humedad (Bolós et al., 1992). De este modo, las vertientes de umbría, ubicadas en la sombra orográfica, presentan menor termicidad y mayor humedad; en consecuencia, una vegetación mucho más frondosa que las de solana y esto no deja de ser cierto en el ámbito de estudio, donde las orientaciones norte (umbría) pueden tener los mismos tipos de vegetación que las sur y no obstante se presentan más desarrolladas o frondosas. 
Tabla 5. Normalización de calidad visual según tipo y estrato de vegetación

\begin{tabular}{|c|c|c|}
\hline Tipo de vegetación & Estratos de vegetación & Valor de Calidad Visual \\
\hline Agrícola con vegetación natural & 2 & 4 \\
\hline Bosques de coníferas & 4 & 10 \\
\hline Bosques de frondosas & 4 & 8 \\
\hline Bosques mixtos & 4 & 8 \\
\hline Espacios con escasa vegetación & 1 & 4 \\
\hline Matorral boscoso de transición & 2 & 5 \\
\hline Matorral esclerófilo & 2 & 7 \\
\hline Pastizales supraforestales naturales & 2 & 4 \\
\hline Prados y praderas & 1 & 6 \\
\hline Sistemas agroforestales adehesados & 3 & No data \\
\hline No data (sin datos) & No data & \\
\hline
\end{tabular}

Fuente: Elaboración propia según el criterio de normalización de Serrada (2008)

Esta consideración encuentra su desarrollo metodológico a raíz de las informaciones digitales sobre el terreno que ofrece el modelo MDE a escala 1:25.000. El trabajo de gestión del territorio contenido en el modelo de elevaciones, y operado a través de herramientas SIG, permitió conocer cuáles son las orientaciones del área, las cuales se reclasificaron siguiendo el siguiente modelo de normalización. Para lograr introducir esta característica de la vegetación en el modelo de evaluación, se optó por obtener una capa a partir del MDE con la función específica de orientación del ArcGis. El resultado es una capa con valores que van de 0 a 360, correspondientes a orientaciones en grados respecto al norte. Ésta se reclasificó (Tabla 6) para obtener una capa con sólo las orientaciones norte; asignando valor 0 a todo lo que no tuviera orientación norte, considerando las orientaciones norte como aquellas que van desde el NO franco hasta el NE franco $\left(337,5^{\circ}-22,5^{\circ}\right)$, pasando por el $\mathrm{N}\left(0^{\circ}\right)$.

Tabla 6. Normalización de la calidad visual según orientaciones de la superficie terrestre

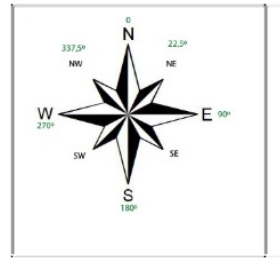

\begin{tabular}{|c|c|}
\hline Valor Orientación & $\begin{array}{c}\text { Valor de } \\
\text { Calidad Visual }\end{array}$ \\
\hline $0-22,5(\mathrm{NNE})$ & 5 \\
\hline $337,5-360(\mathrm{E}, \mathrm{O}, \mathrm{S}, \mathrm{SE}, \mathrm{SO})$ & 0 \\
\hline $22,5-337,5(\mathrm{NNO})$ & 5 \\
\hline
\end{tabular}

Fuente: Elaboración propia según el criterio de normalización de Bolós et al (1992) 
Figura 8. Calidad visual según orientaciones de la superficie con respecto al sol.

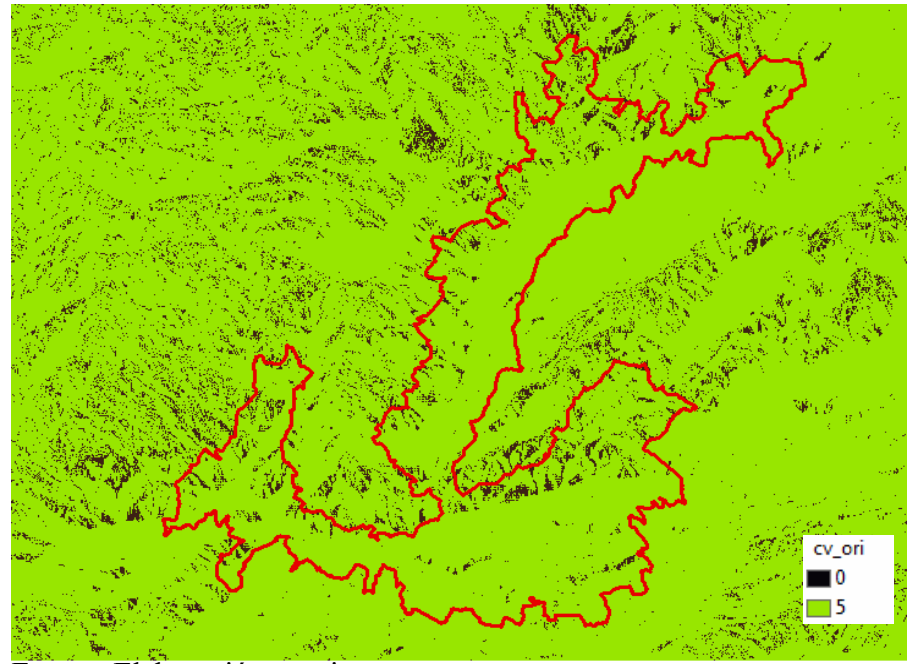

Fuente: Elaboración propia.

Del proceso de normalización se pudo extrapolar la información sobre la calidad visual emanada de las orientaciones, siempre según el modelo expuesto por Serrada (2008). Dicha información se expresó en una cartografía (Figura 8), cuya información está vinculada directamente con el proceso de reclasificación de las orientaciones según la Tabla 6 . Una vez se han conocido y reclasificado según su calidad las orientaciones de la vegetación del área de la Sierra de Guadarrama, la segunda de las fases metodológicas para hallar la calidad de la frondosidad de la vegetación pasa por una ponderación positiva sobre las capas de vegetación natural sobre las no naturales (Singh et al., 2018). Bajo esta consideración, y tomando como referencia la investigación comentada, para tener la frondosidad de la vegetación se utilizó la capa de usos del suelo, con el objeto de enmascarar lo que no corresponde a vegetación natural, obteniendo un raster binomial, donde lo que no fuera vegetación quedara con valor 0 y la vegetación natural con valor 1 (Tabla 7).

Tabla 7. Normalización de la calidad visual según naturalidad de los usos del suelo

\begin{tabular}{|c|c|}
\hline Uso del suelo (CLC11) & Valor Calidad Visual \\
\hline Vegetación natural & 1 \\
\hline $\begin{array}{c}\text { Vegetación no natural, cultivos u otros aprove- } \\
\text { chamientos antrópicos }\end{array}$ & 0 \\
\hline
\end{tabular}

Fuente: Elaboración propia

Tras el proceso de normalización se procede a reclasificar la capa de información sobre el espacio dominado por vegetación natural y por usos que no son éstos. De este modo se consigue generar un mapa de la calidad visual del factor frondosidad (Figura 9). La información de esta capa se multiplicó con la capa de orientaciones 
reclasificada utilizando la Calculadora raster, consiguiendo de esta manera una capa de calidad visual de la vegetación de umbría, que en esta teoría se corresponde a la de mayor frondosidad y que por tanto le confiere una mejor cualidad al paisaje en el que se encuentre.

Figura 9. Calidad visual según la frondosidad

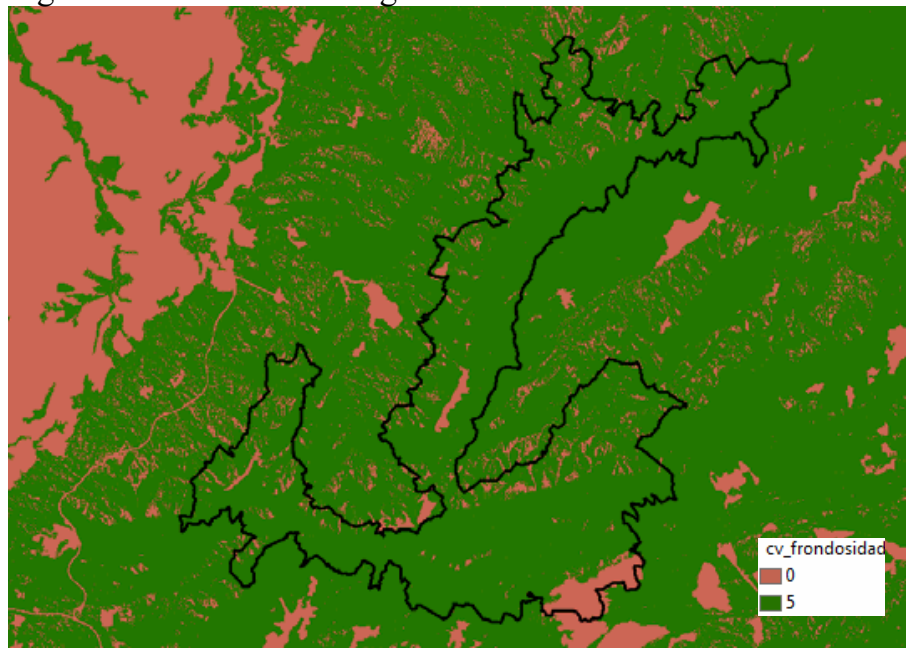

Fuente: Elaboración propia según modelo AHP

\subsubsection{Usos del suelo}

Nuevamente se quiere incidir en la idea que afirma que, entre los elementos que le dan carácter a los paisajes, están los de origen antrópico. Es por eso por lo que se han tenido en consideración en este apartado los usos del suelo, ya que en la actualidad constituyen un verdadero rara avis los espacios en los que la influencia o presencia humana no esté presente $\mathrm{y}$, por ende, los paisajes no se encuentren modificados en un sentido u otro por acción directa o indirecta del hombre (Bolós et al., 1992). Siguiendo esta misma línea argumental, así como las directrices encontradas en la bibliografía sobre la valoración de la calidad visual de los usos del suelo, se ha decidido valorar en este trabajo los factores uso del suelo y diversidad de uso, por tener el territorio unos usos del suelo que además representan elementos importantes de la cultura del lugar, como son los vestigios culturales y las formas de explotación tradicionales, que le confieren su particularidad (Raymond et al, 2010).

A través de la información sobre usos del suelo que ofrece el Corine Land Cover11, el trabajo de investigación pretende conseguir únicamente que queden expresados en el territorio aquellos usos que forman parte del denominado mosaico tradicional de usos (Tello, Garrabou, Cussó y Olarieta, 2008), que en el espacio de la sierra de Guadarrama (Figura 10) se concretan en los territorios en los que se asientan 
los bosques de coníferas, la presencia de matorral mediterráneo esclerófilo, los pastizales supraforestales naturales y los sistemas agroforestales adehesados. Detrás de esta premisa no se parte de la idea de que todo uso tradicional es positivo de cara a la valoración paisajística y que cualquier uso moderno, por el contrario, es lesivo, sino que más bien se prima en la valoración la existencia de usos históricos en la configuración de paisajes, como el mediterráneo que centra esta investigación, de honda raíz antrópica.

Figura 10. Usos tradicionales del suelo en el área de la Sierra de Guadarrama

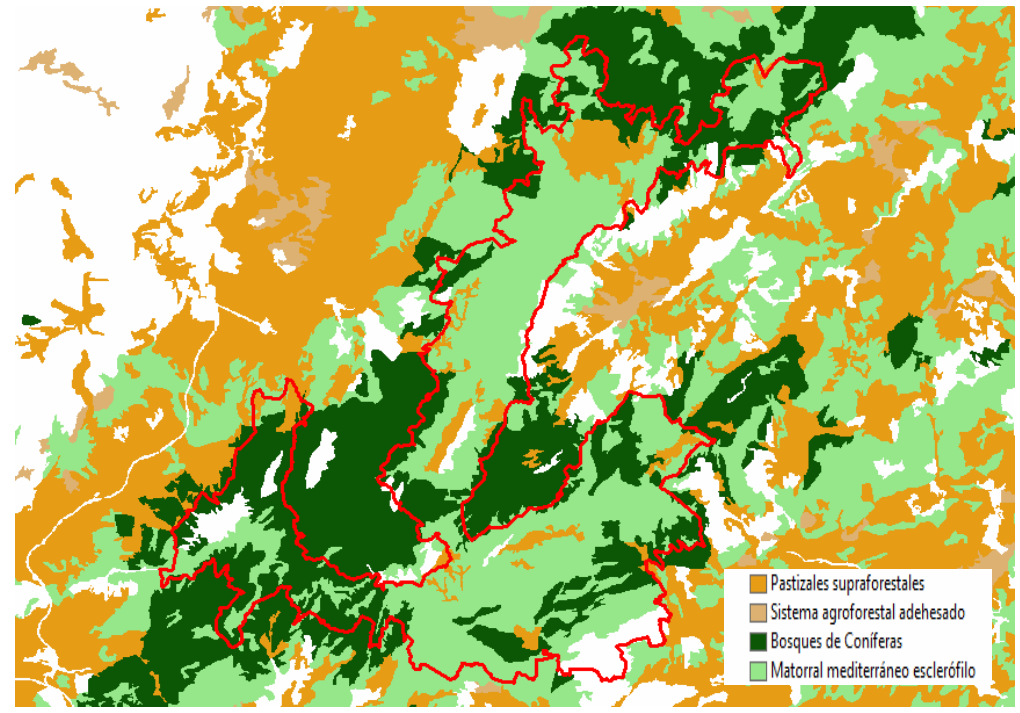

Fuente: Elaboración propia

Para el tratamiento de este factor general, se empleó la citada base de datos del Corine Land Cover11; desde el formato shp. se exportó a raster desde el propio hardware ArcGis, de la misma manera que en el caso del mapa de hábitat usado para la vegetación. Al valorar los usos del suelo y reclasificar según las puntuaciones de la calidad visual (Tabla 8), se tuvo cuidado de asignar valor 0 a todo lo que fuera NO DATA. 
Tabla 8. Reclasificación de la calidad visual según los usos del suelo

\begin{tabular}{|c|c|}
\hline Tipo de uso del suelo CLC12 & Valor de Calidad Visual \\
\hline Extracción minerales & 0 \\
\hline Zonas quemadas & 0 \\
\hline Zonas industriales & 1 \\
\hline Infraestructuras viales & 1 \\
\hline Espacios con escasa vegetación & 1 \\
\hline Tejido urbano continuo & 5 \\
\hline Turberas y prados turbosos & 5 \\
\hline Matorrales húmedos & 6 \\
\hline Roquedo & 7 \\
\hline Matorral transición & 7 \\
\hline Agrícola veg. natural & 7 \\
\hline Prados y praderas & 8 \\
\hline Mosaico de cultivos & 8 \\
\hline Láminas de agua & 8 \\
\hline Pastizales naturales & 9 \\
\hline Bosque mixto & 10 \\
\hline Bosque de frondosas & 10 \\
\hline Bosque de coníferas & 10 \\
\hline
\end{tabular}

Fuente: Elaboración propia según el criterio de normalización de Vallina (2017)

Una vez valorado el mosaico de usos tradicionales presente en el ámbito de estudio, se hace necesario el análisis de la incidencia de otro factor de capital influencia en la calidad visual de un determinado paisaje, la diversidad de usos. Este factor pondera positivamente aquellas áreas donde se mezclan distintos tipos de cubiertas, como cultivos, con áreas de vegetación natural o zonas protegidas, formando un mosaico de usos que visualmente resulta muy atractivo, sobre todo en una región donde los usos tradicionales, como los pastizales, han cobrado una mayor prevalencia en el imaginario común desde hace décadas. Así, la diversidad de uso se consideró como un factor que le imprime un carácter particular al paisaje del territorio y ha sido valorada de modo que a mayor diversidad o entremezclado de cubiertas del suelo, mayor calidad visual del paisaje (Figura 11). Para ello, se reclasificó la capa de usos del suelo, de modo que sólo quedaran representados aquellos usos que se corresponden al mosaico mediterráneo de montaña (bosques de coníferas + praderas natural + matorral esclerófilo + sistemas adehesados), manteniendo sus atributos. Al resto de usos se les asignó el valor de No Data para que no los contabilizara en la operación. Se aplicó entonces un análisis de vecindad, denominado Estadística Focalizada, que calculo la variedad de usos en ventanas móviles de 13 x 13 celdas, arrojando como resultado el número de usos diferentes para cada ventana móvil. 
Figura 11. Calidad visual del paisaje de mosaico mediterráneo de montaña

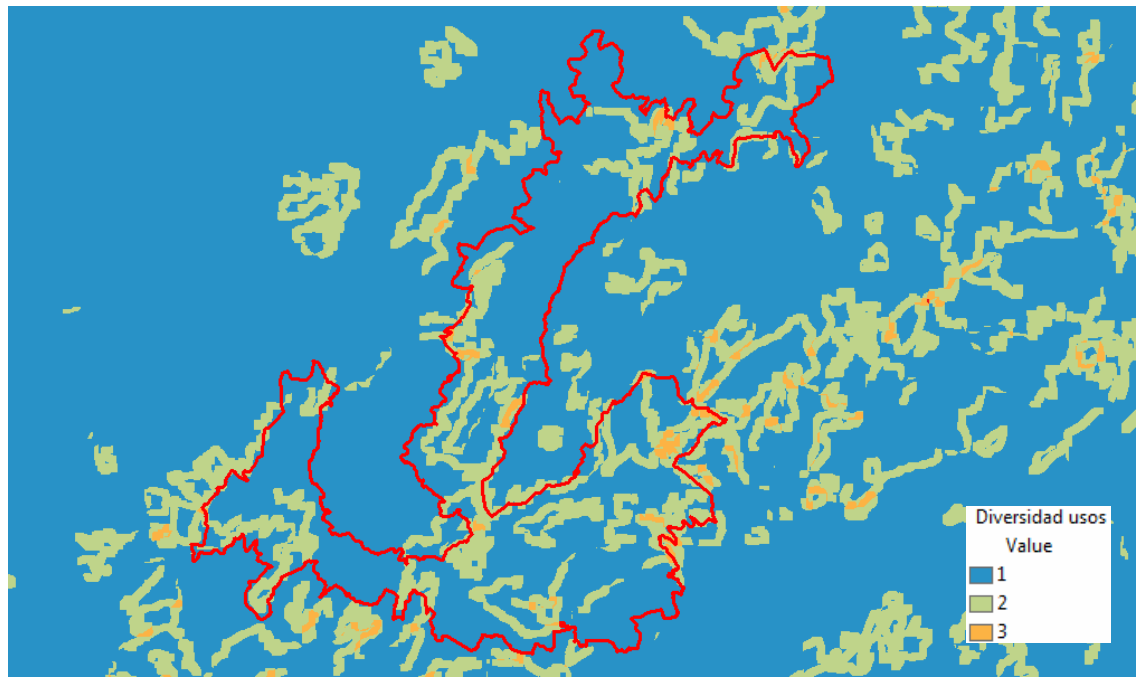

Fuente: Elaboración propia

\subsubsection{Elementos singulares}

Entre los elementos que conforman los valores considerados como intrínsecos en el paisaje de la Sierra de Guadarrama, el presente trabajo de investigación pone el acento en la incidencia visual y perceptiva holística que tienen determinadas piezas del paisaje, tales como los prados y pastizales naturales o los roquedos, ambos componentes indisolubles a la idea de paisaje de alta montaña mediterránea que encarna el Guadarrama. Toda vez que ya han sido evaluada, en cierto modo, la presencia de este tipo de usos y aprovechamientos del suelo dentro del ámbito de estudio, desde aquí se propone concederles una cierta predominancia frente a otros valores, tanto por su especial trascendencia visual, como por los valores culturales, sociales y humanos que tradicionalmente se han vinculado tanto a los pastizales del altura como a las formaciones de roca desnuda, ambos tan característicos del sector serrano que aquí se analiza.

Para el tratamiento de estos factores y su posterior conversión en valores de calidad visual, primeramente se ha necesitado descargar las capas. En el caso de los pastizales y prados se ha utilizado la capa de cubiertas del Corine Land Cover 11, al igual que en el caso de los roquedos.

La base de la metodología de valoración de los elementos singulares trata de vincular el número de elementos vistos con la calidad visual percibida (Tabla 9), todo ello siempre considerando la distancia entre observador y elemento visual como un atenuador de las calidades. De este modo, en la tabla se puede apreciar la normalización propuesta según el número de elementos singulares percibidos por un observador cualquiera, la distancia entre ambos, y la calidad visual estimada. 
Tabla 9. Normalización de la calidad visual en función de los elementos vistos/distancia

\begin{tabular}{|c|c|c|}
\hline $\begin{array}{c}\text { Número elementos } \\
\text { vistos }\end{array}$ & $\begin{array}{c}\text { Distancia elemento- } \\
\text { observador (metros) }\end{array}$ & $\begin{array}{c}\text { Valor de Calidad } \\
\text { Visual }\end{array}$ \\
\hline 0 & 500 & 0 \\
$1-3$ & 500 & 7 \\
$3-8$ & 500 & 8 \\
$8-10$ & 500 & 9 \\
$>10$ & 500 & 10 \\
\hline 0 & 1000 & 0 \\
$1-3$ & 1000 & 6 \\
$3-8$ & 1000 & 7 \\
$8-10$ & 1000 & 8 \\
$>10$ & 1000 & 9 \\
\hline 0 & 2000 & 0 \\
$1-3$ & 2000 & 5 \\
$3-8$ & 2000 & 6 \\
$8-10$ & 2000 & 7 \\
$>10$ & 2000 & 8 \\
\hline 0 & 3000 & 0 \\
$1-3$ & 3000 & 5 \\
$3-8$ & 3000 & 6 \\
$8-10$ & 3000 & 6 \\
$>10$ & 3000 & 7 \\
\hline
\end{tabular}

Fuente: Elaboración propia según el criterio de normalización de Vallina (2017)

Bajo estas premisas el primero de los pasos que debe darse es extraer de la capa de usos Corine Land Cover11 la información geográfica referida a los prados y pastizales supraforestales naturales y a los prados y praderas naturales, así como la relativa a los roquedos y canchales del ámbito. Una vez estas dos clases de elementos han quedado como entes separados del resto de usos se procede a realizar dos estudios de proximidad, mediante la generación de anillos múltiples buffer, manteniendo para ello las distancias consideradas como adecuadas para la visualización correcta y clara de los elementos, en una franja de distancia que va desde los 500 hasta los 3000 metros (Figuras 12 y 13). Una vez realizados los estudios de proximidad de los dos tipos de elementos para conocer hasta dónde llegan sus respectivas incidencias visuales se procede a analizar sus cuencas visuales, es decir, desde dónde pueden ser vistas por el hombre, para lo cual se aplica un factor de corrección a 1,8 metros sobre el suelo que simula la estatura media de la población (Serrano, 2015). A sabiendas que los conceptos de incidencia y cuenca visuales no son directamente equiparables, la presente investigación procede a homogeneizar su significado, muy concretamente en cuando al valor de la distancia desde la cual son visibles los objetos, con el objetivo de no introducir mayor complejidad al establecimiento del modelo de evaluación. De igual modo, se aclara que el factor de corrección de 1,8 metros es un valor usado internacionalmente, que aglutina el valor 
medio de altura de observadores hombres y mujeres usado convencionalmente. La presente investigación carece de juicio formado para establecer si la media de la altura de los observadores que visitan y disfrutan el Parque Nacional de la Sierra de Guadarrama tienen esa media o es inferior, por lo que se ha optado por la utilización del valor de convención internacional.

Figura 12. Estudio de proximidad y estimación de calidad visual de los prados y pastizales naturales
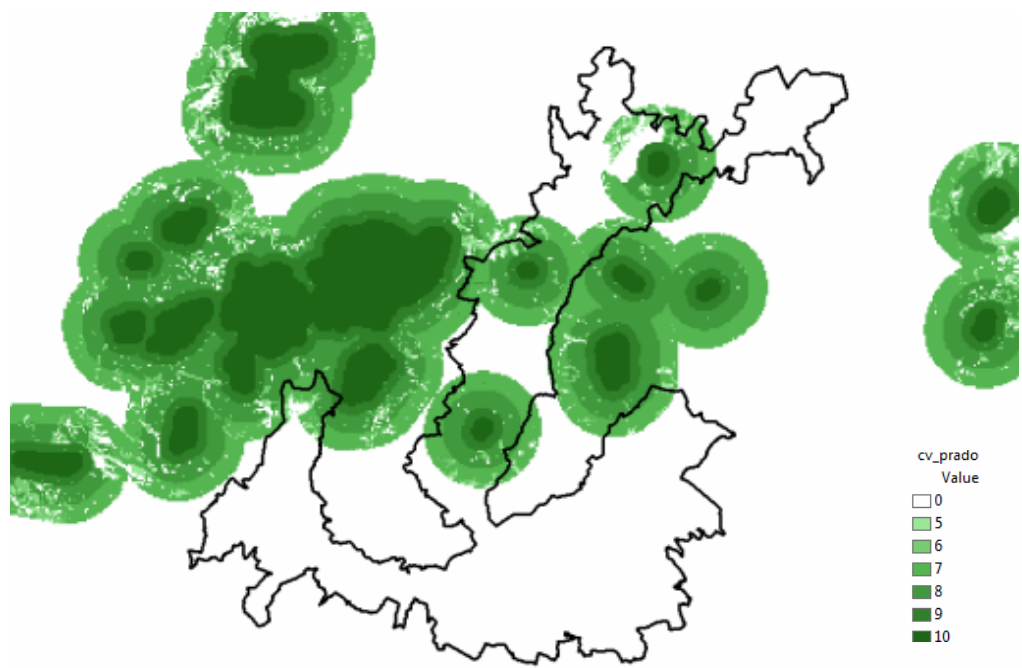

Fuente: Elaboración propia según modelo AHP

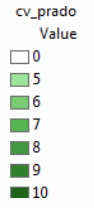

Figura 13. Estudio de proximidad y estimación de calidad visual de los roquedos

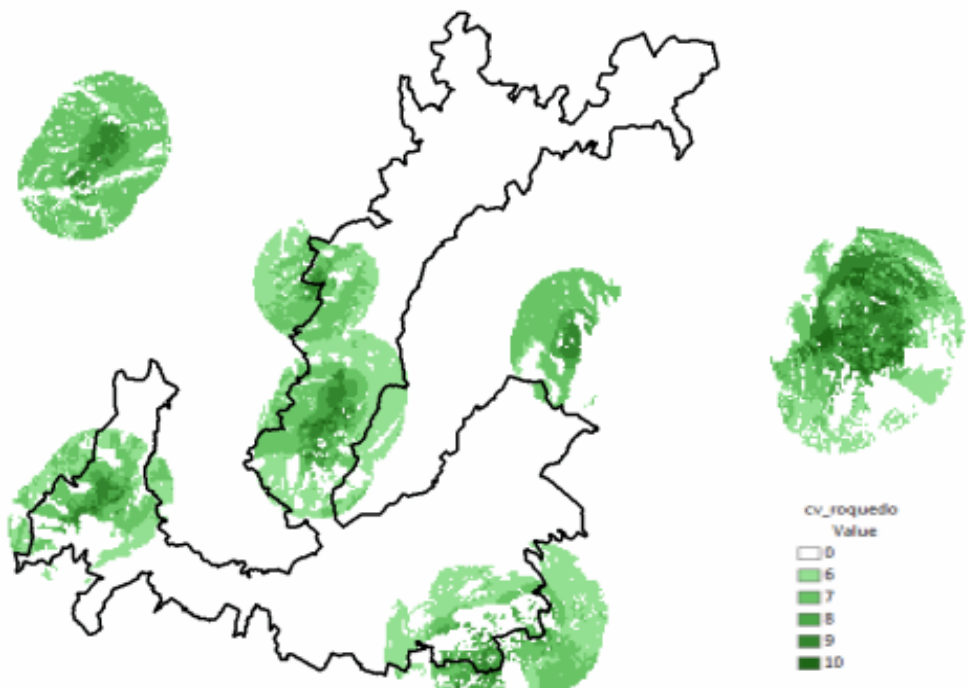

Fuente: Elaboración propia según modelo AHP 
Del resultado de la combinación mediante el SIG de las capas de proximidad y cuencas visuales se extraerá unas capas de atributos, las cuales se han de normalizar según los valores estipulados en la Tabla 9, de tal modo que se extraiga información legible sobre la calidad visual individual de cada uno de los elementos singulares analizados.

\subsection{Ponderación de los criterios y aplicación del algoritmo EMC}

Cuando todos los valores intrínsecos del paisaje objeto de esta investigación han sido evaluados según sus indicadores determinados, la labor del investigador consiste en discernir cuáles de estos criterios y componentes tienen predominancia sobre otros desde el punto de vista visual, ya que no todos tendrán la misma significancia/relevancia visual en el conjunto de la imagen paisaje analizada. Es en este punto donde surge la necesidad de clarificar, de un modo lo más sistemático y objetivo posible, de qué modo pasarán a ponderarse cada uno de los criterios y componentes del paisaje contemplados en el modelo de valoración. La ponderación de los criterios se define como el peso o importancia que se le atribuye a cada uno de los factores utilizados para el cálculo de la fragilidad visual del paisaje dentro del modelo diseñado para ello.

La valoración sobre la fragilidad visual del paisaje relativo al Parque Nacional del Guadarrama estará supeditada, de forma indefectible, a la asignación de ponderaciones que se le aporte, realizada ésta mediante la utilización del método Analytic Hierarchy Process (AHP) o Método Analítico Jerárquico. Este método se caracteriza porque descompone y organiza el problema de forma visual en una estructura jerárquica. El método establece la importancia relativa de los elementos de cada jerarquía a partir de la matriz de comparación por pares de Saaty (1980), una metodología no de valoración, sino de toma de decisiones, aceptada internacionalmente y que es usada en valoraciones en todo tipo de disciplinas. A cada par de variables se le asigna un valor de la escala entre 1/9 (extremadamente menos importante el primer criterio respecto al segundo) hasta 9 (extremadamente más importante el primer criterio respecto al segundo) (Gráfico 2).

Gráfico 2. Escala de medida en las comparaciones entre factores.

\begin{tabular}{ccccccccc}
$1 / 9$ & $1 / 7$ & $1 / 5$ & $1 / 3$ & 1 & 3 & 5 & 7 & 9 \\
\hline extrema & fuerte & & moderada & igual & moderada & fuerte & extrema \\
menos importante & & & & & & & & más importante
\end{tabular}

Fuente: Elaboración a partir de Saaty (1980)

En la primera matriz se asignan los valores a cada par de variables que la componen. Por ejemplo, la fisiografía es fuertemente más importante que la vegetación natural de modo que su valor es de 7. Dado que las comparaciones son 
recíprocas, la vegetación natural será fuertemente menos importante que la fisiografía (1/7). En la segunda matriz se han calculado los valores de las fracciones y éstos se han sumado columna a columna. La tercera matriz es la matriz normalizada a suma 1 , donde cada valor de una columna se divide por la suma de los valores de esa misma columna. Posteriormente se desarrolla el vector de prioridad de cada criterio calculando el promedio de cada fila de la matriz normalizada. Como resultado de este proceso se obtiene el peso de cada criterio (Zube, 1984). 
Tablas 10,11 y 12 . Pesos de la calidad intrínseca calculados mediante la matriz de Saaty

\begin{tabular}{|c|c|c|c|c|}
\cline { 2 - 5 } \multicolumn{1}{c|}{} & Fisiografía & $\begin{array}{c}\text { Vegetación } \\
\text { natural }\end{array}$ & $\begin{array}{c}\text { Usos del } \\
\text { suelo }\end{array}$ & $\begin{array}{c}\text { Elementos } \\
\text { singulares }\end{array}$ \\
\hline Fisiografía & 1 & 1 & 9 & 1 \\
\hline Vegetación natural & 1 & 1 & 7 & 7 \\
\hline Usos del suelo & $1 / 9$ & $1 / 7$ & 1 & 7 \\
\hline Elementos singulares & 1 & $1 / 7$ & $1 / 7$ & 1 \\
\hline
\end{tabular}

\begin{tabular}{|c|c|c|c|c|}
\cline { 2 - 5 } \multicolumn{1}{c|}{} & Fisiografía & $\begin{array}{c}\text { Vegetación } \\
\text { natural }\end{array}$ & $\begin{array}{c}\text { Usos del } \\
\text { suelo }\end{array}$ & $\begin{array}{c}\text { Elementos } \\
\text { singulares }\end{array}$ \\
\hline Fisiografía & 1.00 & 1.00 & 9.00 & 1.00 \\
\hline Vegetación natural & 1.00 & 1.00 & 7.00 & 7.00 \\
\hline Usos del suelo & 0.11 & 0.14 & 1.00 & 7.00 \\
\hline Elementos singulares & 1.00 & 0.14 & 0.14 & 1.00 \\
\hline
\end{tabular}

\begin{tabular}{|c|c|c|c|c|c|}
\cline { 2 - 6 } \multicolumn{1}{c|}{} & Fisiografía & $\begin{array}{c}\text { Vegetación } \\
\text { natural }\end{array}$ & $\begin{array}{c}\text { Usos del } \\
\text { suelo }\end{array}$ & $\begin{array}{c}\text { Elementos } \\
\text { singulares }\end{array}$ & Peso (\%) \\
\hline Fisiografía & 0.32 & 0.44 & 0.53 & 0.07 & 40 \\
\hline Vegetación natural & 0.32 & 0.44 & 0.40 & 0.43 & 25 \\
\hline Usos del suelo & 0.04 & 0.06 & 0.05 & 0.07 & 20 \\
\hline Elementos singulares & 0.32 & 0.06 & 0.02 & 0.06 & 15 \\
\hline
\end{tabular}

Fuente: Elaboración propia

Según las determinaciones del AHP, una vez se ha procedido a la normalización de los factores mencionada en las líneas anteriores se calculan los pesos porcentuales de los mismos, con el objetivo de concluir con una integración de las capas de información de cada criterio a partir de la aplicación de un algoritmo de EMC. La metodología EMC se sirve, entre otras, de las técnicas compensatorias, basadas en el precepto de que un valor alto de una alternativa en un factor puede compensar un valor bajo de la misma alternativa en otro factor. La Sumatoria Lineal Ponderada, como una de las técnicas EMC compensatorias, será la utilizada en este trabajo (Gómez y Barredo, 2005). Esta técnica procede asignando el valor en cada componente como resultado de la función de los componentes del nivel inferior que en él confluyen, La fórmula de la Sumatoria Lineal Ponderada es: $\mathrm{R}=\Sigma \mathrm{w}^{*} \mathrm{v}$

Donde (r) es el nivel de adecuación de la alternativa, (w) es el peso del criterio y (v) es el valor normalizado del factor. Como puede apreciarse en la figura 3, en el 
primer nivel de los dos grandes componentes de la fragilidad visual del paisaje se han estimado unas ponderaciones del $60 \%$ en el componente relativo a la fragilidad visual intrínseca, es decir, que los valores consustanciales del paisaje, no vinculados ni a la acción del hombre en el territorio ni a su papel como observador del territorio, tienen una influencia sobresaliente en el modelo de fragilidad sobre el cual pivota la presente investigación. Ello viene a reforzar la tesis de que buena parte de la calidad visual que pueda ostentar un determinado paisaje están vinculados fundamentalmente a las realidades fisiográficas y de dominancia de usos del suelo en el mismo.

El $40 \%$ restante de la ponderación del modelo de calidad visual confeccionado atañe a los componentes visuales adquiridos del paisaje, ligados a la acción del hombre y a su percepción como observador del paisaje. Dentro de este gran componente de la calidad visual queda patente que las principales determinaciones de este valor vienen dadas desde los factores de observación fija del territorio, debido a su permanencia y visibilidad en el ámbito guadarrameño.

\subsubsection{Superposiciones ponderadas de los criterios de evaluación}

Por último, una vez obtenidas las capas resultantes de la normalización de valores tanto de las pendientes como de los desniveles observables en el área de estudio, se procede a realizar una operación de superposición ponderada sobre ambos descriptores del elemento fisiografía. En la toma de decisiones sobre la ponderación dada a los valores fisiográficos esta investigación se ha basado en la técnica EMC. La capa resultante de la superposición aportará el valor de calidad visual según la fisiografía en el entorno de la Sierra de Guadarrama (Figura 14).

Figura 14. Calidad visual del criterio fisiografía
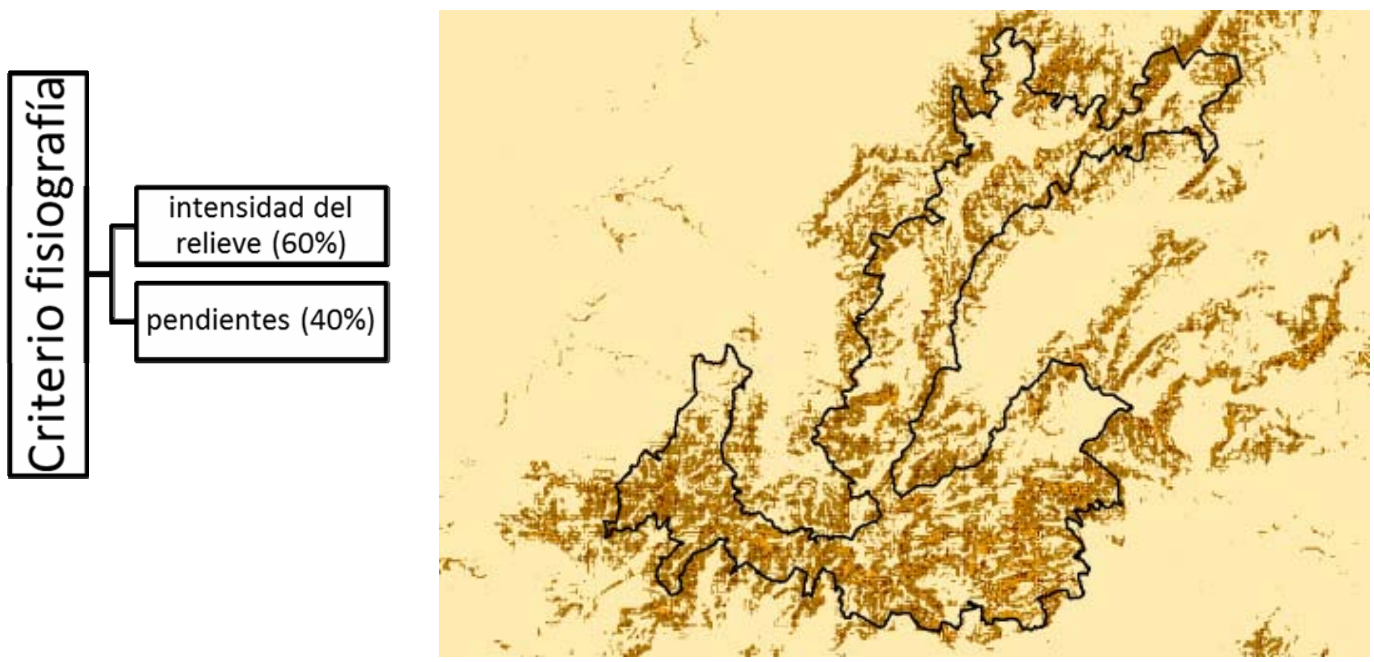

Fuente: Elaboración propia tras aplicación métodoAHP 
En cuanto a la agregación de valores obtenidos acerca del criterio vegetación natural, la ECM aplicada al proceso ha determinado los pesos específicos de las variables analizadas. La herramienta de superposición ponderada de la herramienta SIG posteriormente construyó la capa de calidad visual según la vegetación natural (Figura 15).

Figura 15. Calidad visual del criterio vegetación
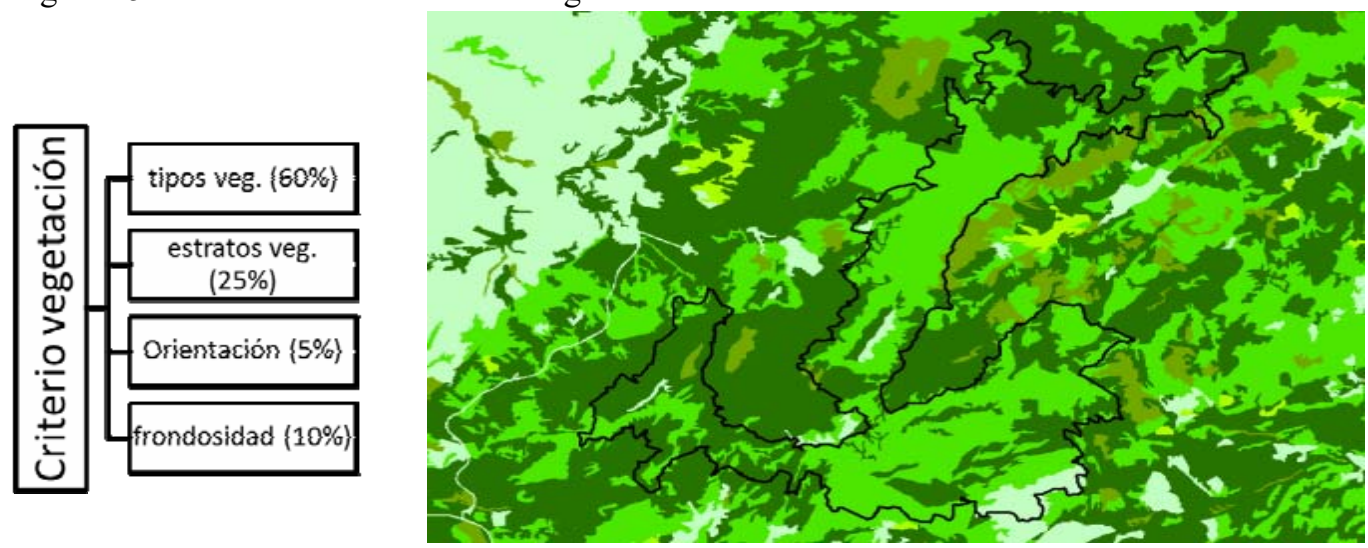

Fuente: Elaboración propia tras aplicación métodoAHP

El proceso de evaluación multicriterio aplicado al criterio de usos del suelo ha arrojado valiosa información acerca de la situación de los valores de calidad visual del paisaje de la Sierra de Guadarrama según los usos del suelo determinados (Figura 16).

Figura 16. Calidad visual del criterio usos del suelo
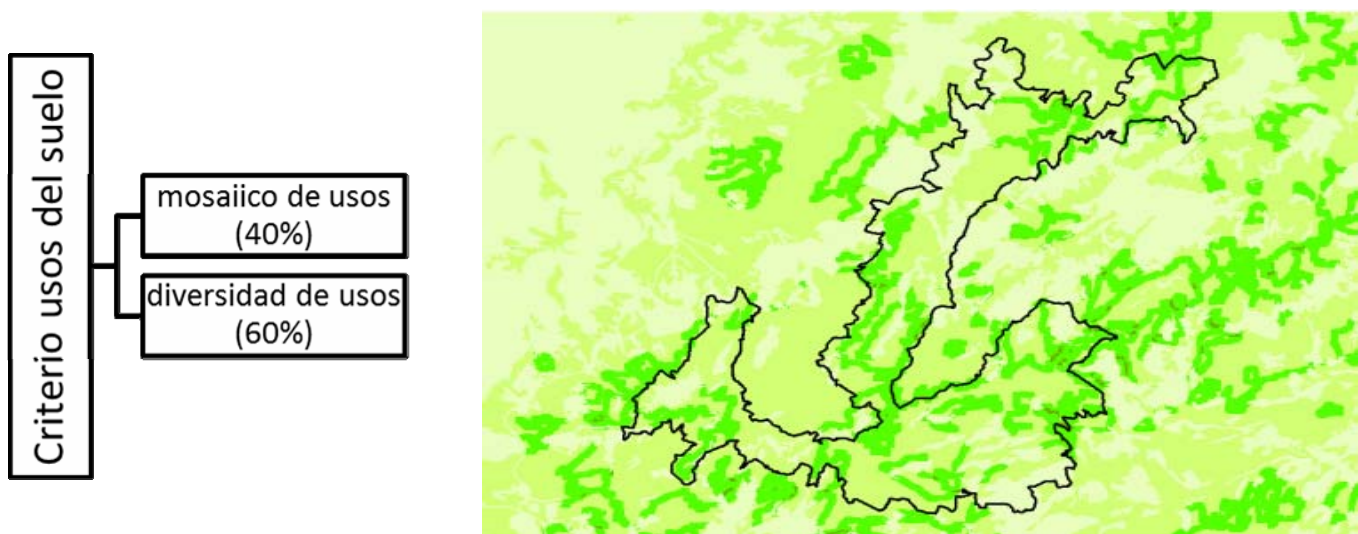

Fuente: Elaboración propia tras aplicación métodoAHP 
Por último, queda por construir la capa resultante de la superposición ponderada elaborada a partir de las determinaciones de la AHP para las variables referidas a la calidad visual de los elementos singulares que alberga el Parque Nacional de la Sierra de Guadarrama (Figura 17).

Figura 17. Calidad visual del criterio elementos singulares
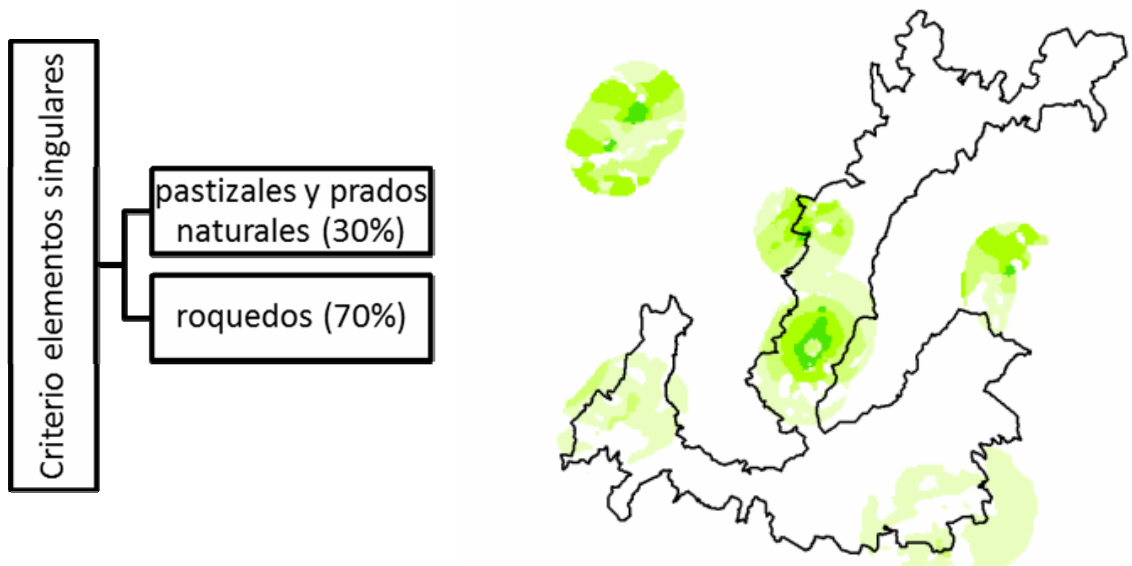

Fuente: Elaboración propia tras aplicación métodoAHP

\section{Resultados y conclusiones}

Producto de la aplicación del modelo de calidad visual específico para los valores de calidad intrínseca se elaboró, como parte culminante de esta aproximación metodológica, la superposición ponderada de los cuatro criterios estudiados, de modo que pudiera construirse una cartografía del Parque Nacional de la Sierra de Guadarrama y su área aledaña según los criterios innatos de su paisaje. En dicha cartografía quedó expresada la calidad visual del paisaje de la zona mediante una escala de valor que, después de ser normalizada y reclasificada para hacer más legible el mapa resultante, muestra una escala de cuatro niveles de calidad visual, siendo el primero el de una valoración más baja, y el cuatro el más elevado (Figura 18). 
Figura 18. Mapa de la calidad visual del Parque Nacional de la Sierra de Guadarrama según los valores de calidad intrínseca del paisaje

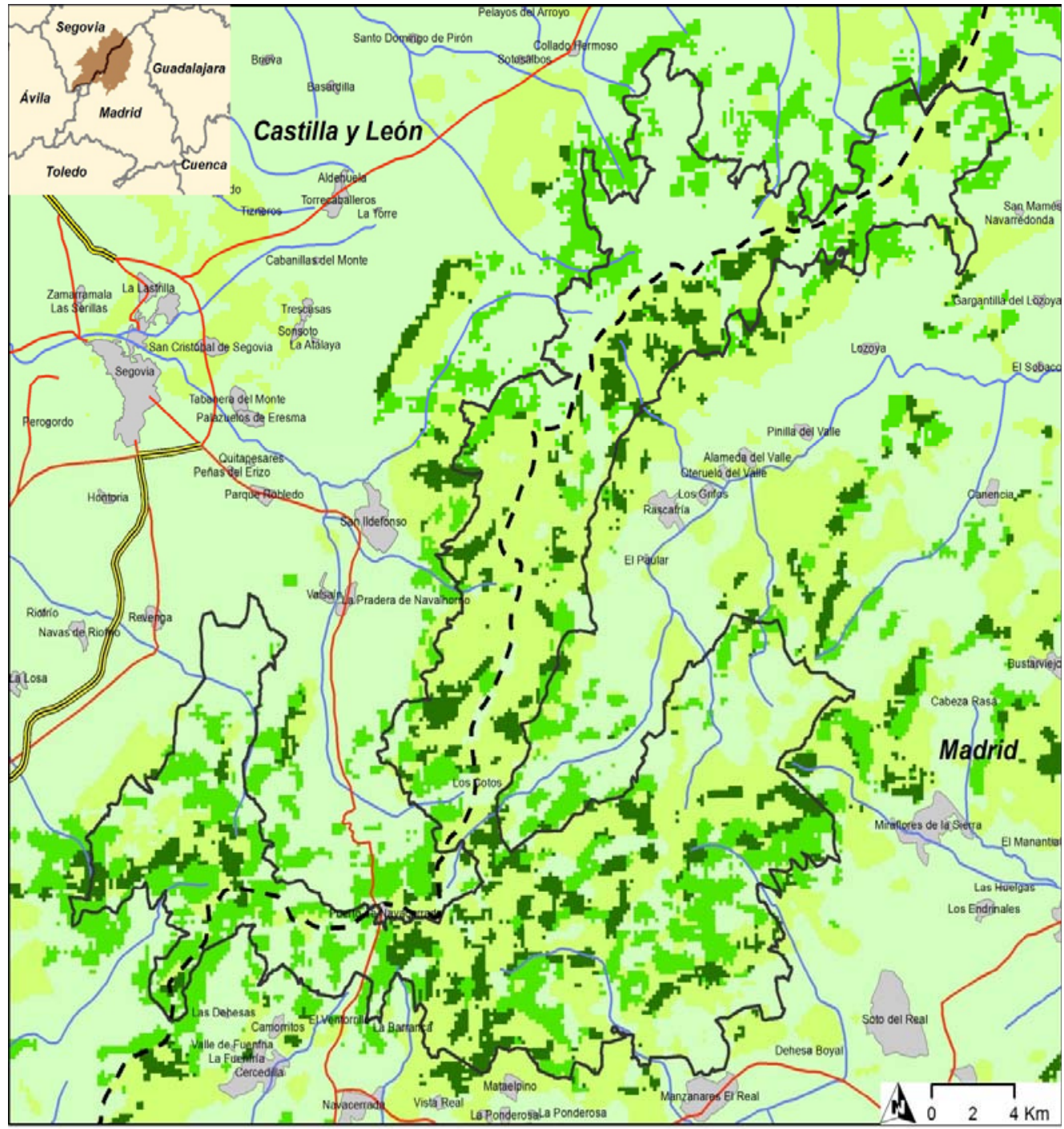

Calidad Visual del Paisaje. Valores intrinsecos
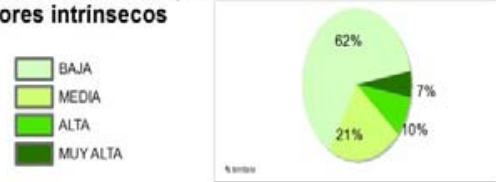

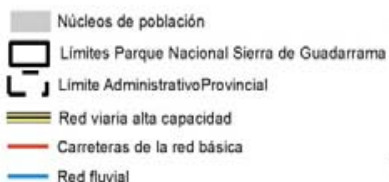

Laboratorio Geografia Aplicada Universidad Autónoma de Madrid

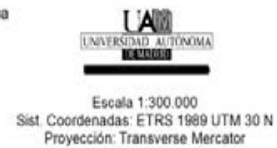

Fuente: Elaboración propia. 
Desde el punto de vista de la aplicación, se demuestra una vez más que tanto los estudios de calidad como los de fragilidad visual del paisaje son fundamentales para una correcta ordenación del territorio, especialmente en espacios naturales en los que es prioritaria la política de conservación de la naturaleza, y en aquellos en los que son compatibles usos como las actividades turístico-recreativas o el uso público con la conservación de los recursos naturales. Es fundamental señalar que los cuatro valores de calidad expresados en el mapa se refieren a calidad visual intrínseca del ámbito de estudio de Guadarrama, por lo tanto, no son valores de validez universal.

Sin duda estudios como este, vinculados a la percepción del paisaje, proporcionan información para disminuir la presión humana sobre determinados sitios de gran valor ecológico donde se concentra tradicionalmente el uso público, indicando parajes alternativos con una elevada atracción (Tabla 13). En este sentido, la calidad visual del paisaje, junto a otros recursos, es frecuentemente empleada como criterio para señalar zonas que deben ser preservadas frente a actuaciones que suponen un cierto impacto ambiental.

Tabla 13. Distribución de las categorías de calidad visual de los elementos intrínsecos del paisaje

\begin{tabular}{|c|c|c|}
\hline CATEGORÍA & SUPERFICIE (HA) & $\mathbf{\%}$ \\
\hline Baja & 117.773 & 62 \\
\hline Media & 39.891 & 21 \\
\hline Alta & 18.995 & 10 \\
\hline Muy alta & 13.297 & 7 \\
\hline TOTAL & $\mathbf{1 8 9 9 5 7}$ & $\mathbf{1 0 0}$ \\
\hline
\end{tabular}

Fuente: Elaboración propia

En la actualidad es cierto que la inmensa mayoría de técnicas de evaluación de la calidad visual del paisaje son criticadas por su subjetividad, por el reducido y característico ámbito territorial o de conocimiento al que son aplicadas. Esta investigación ya partía de la premisa de que no existe una técnica de valoración del paisaje correcta que excluya a las demás y que la mayor parte de los trabajos se basan en métodos subjetivos pero sistemáticos. La parcialidad con respecto a la valoración del paisaje se hace aún más patente si cabe cuando variables de tipo estético y cultural, relacionadas con la percepción humana, entran a formar parte de la ecuación de la calidad/fragilidad visual. El desarrollo de la metodología indirecta de evaluación que aquí se presenta tiene un claro sesgo vinculado a la relevancia y grado de 
participación de los parámetros que constituyen su modelo. Dicho sesgo está indiscutiblemente asociado a la dirección y elección de los componentes del paisaje estudiados y los enfoques de fragilidad aportados. Únicamente mediante la exigencia de un mayor y más certero conocimiento del medio objeto de estudio por parte del equipo gestor y una incorporación más exhaustiva de parámetros paisajísticos y la importancia relativa dada por medio de las ponderaciones realizadas aportará una solución eficaz capaz de, por lado, ofrecer una alternativa menos sesgada y, por otro, ajustarse más a las exigencias y peculiaridades del complejo espacio analizado.

La propuesta metodológica aquí expuesta nace como aspiración de ser una pauta más que ayude a determinar el valor que presenta el paisaje de un espacio tan concreto como puede ser el ámbito de la Sierra de Guadarrama dentro del Sistema Central Ibérico, pero en todo momento la investigación ha considerado necesaria la elaboración de un completo método de valoración con rangos y criterios bien establecidos, que sea una herramienta más en el campo de la valoración perceptual del paisaje desde el punto de vista de la disciplina geográfica.

\section{Referencias bibliográficas}

Alberruche, M., Arranz, J., Rodríguez, V., Fernández, F., Rodríguez, R., y Vadillo, L. (2015). Methodology for the evaluation of the residual impact on landscape due to an opencast coal mine in Laciana Valley (Spain). DYNA Universidad Nacional de Colombia, 82(190), 60- 69.

Aramburu, M., Escribano, R., López, R., y Sánchez, P. (2005). Cartografía del Paisaje de la Comunidad Autónoma del la Rioja. Logroño: Gobierno de la Rioja-ETSI de Montes UPM.

Bolós, M., et al. (1992). Manual de Ciencia del Paisaje. Barcelona: Masson.

Cáncer, L. (1994). Aproximación critica a las teorias más representativas de la ciencia del paisaje. Geographicalia, 17-34.

Cohen, J., Blinn, C.E., Boyle, K.J. (2016). Hedonic Valuation with Translating Amenities: Mountain Pine Beetles and Host Trees in the Colorado Front Range. Environmental and Resource Economics, 63, 613-642. doi:10.1007/s10640-014-9856-y

Consejo de Europa (2000): Convenio europeo del paisaje. http://www.coe.int/t/dg4/cultureheritage/heritage/landscape/versionsconvention/spanish.pd f]

de la Fuente, G., Atauri, J., \& de Lucio, J. (2004). El aprecio por el paisaje y su utilidad en la conservación de los paisajes de Chile Central. Ecosistemas. Revista científica de ecología y medio ambiente, 13 (2), 82-89.

Delgado, A. y Pantoja, F. (2016). Valoración del paisaje en una propuesta de turismo sostenible: la "Ruta del Oro", Nariño (Colombia). Cuadernos de Geografía: Revista Colombiana de Geografía, 25(1), 233-253.

Díaz, M. y Galiana, F. (1996). Estudio paisajístico de la Huerta de Valencia. Valencia: Ayto. de Valencia. 
Franch- Pardo, I. y Cáncer, L. (2017). El componente visual en la cartografía del paisaje. Aptitud paisajística para la protección en la cuenca del río Chiquito (Morelia, Michoacán). Investigaciones Geográficas, 93, 1-19. DOI: dx.doi.org/10.14350/rig.54730

Galiana, F. y Vallés, M. (2007). La planificación del paisaje en la Comunidad Valenciana: Análisis de la calidad y la fragilidad visual. Saitabi(57), 111-125.

Gómez, M. y Barredo, J. I (2005): Sistemas de información geográfica y evaluación multicriterio en la ordenación del territorio. Madrid, Ed. Ra-Ma. Pp 67- 72.

Hermes, J., Van Berkel, D., Burkhard, B., Plieninger, T., Fagerholm, N., Haaren, C., \& Albert, C. (2018). Assessment and valuation of recreational ecosystem services of landscapes. Ecosystem services, 31, 289-295. doi:10.1016/j.ecoser.2018.04.011

Karanik, M., Wanderer, L., Gomez-Ruiz, J.A., Peláez, J.I. (2016) Reconstruction methods for AHP pairwise matrices: How reliable are they?. Applied Mathematics and Computation, 27. 103-124, https://doi.org/10.1016/j.amc.2016.01.008.

Martín, B. y Otero, I. (2012). Mapping the visual landscape quality in Europe using physical attributes. Journal of Maps, 1(8), 56-61.

Montoya, R., Padilla, J. y Standford, S. (2003). Valoración de la calidad y fragilidad visual del paisaje en el valle de Zapotitlán de las Salinas, Puebla (México). Boletín de la Asociación de Geógrafos Españoles (35), 123-136. Obtenido de http://age.ieg.csic.es/boletin/35/3506.PDF

Panareda, J. (2009). Evolución en la percepción delpaisaje de ribera. Boletín de la AGE (51), 305-324.

Ramos, A. y González, F. (1987): Paisaje, en Ramos Fernández, A. (Coord). Diccionario de la Naturaleza. Hombre, Ecología, paisaje. Madrid. Espasa-Calpe. pp 682-690.

Raymond, C., Fazey, I., Reed, M., Stringer, L., Robinson, G. y Evely, A. (2010). Integrating local and scientific knowledge for environmental management. Journal of Environmental Management, 1766-1777.

Rivera, A., Cruz García, J., Pérez, A., Gallardo, F., \& de la Cruz, M. (2014). La percepción en la evaluación del paisaje. Revista Mexicana de Ciencias Agrícolas, 9, 1811-1817.

Saaty, T.L. (1980): The Analytic Hierarchy Process. New York, McGraw Hill.

Saeidi, S., Mohammadzadeh, M., Salmanmahiny, A., Mirkarimi, S. (2017). Performance evaluation of multiple methods for landscape aesthetic suitability mapping: A comparative study between Multi-Criteria Evaluation, Logistic Regression and Multi-Layer Perceptron $\begin{array}{lllll}\text { neural network. Land Use } & \text { Policy, }\end{array}$ https://doi.org/10.1016/j.landusepol.2017.05.014.

Serrada, R. (2008). Apuntes de Selvicultura. (UPM, Ed.) Madrid: ETSI Ingeniería Forestal.

Serrano, D. (2015). Valoración escénica de paisaje periurbano con utilidad en planeamiento territorial. Estudio de caso en la Región Metropolitana de Barcelona. Investigaciones Geográficas.Boletín del Instituto de Geografía, UNAM, 88, 109-121.

Singh, H., Garg, R.D., Karnatak, H.C., Roy, A. (2018) Spatial landscape model to characterize biological diversity using R statistical computing environment, Journal of Environmental Management,206,1211-1223, doi.org/10.1016/j.jenvman.2017.09.055.

Tello, E., Garrabou, R., Cussó, X. y Olarieta, J. (2008). Una interpretación de los cambios de uso del uselo desde el punto de vista del metabolismo social agrario. La comarca catalana del Vallès, 1853-2004. Revista Iberoamericana de Economía Ecológica, 97-115. 
Tress, B., Tress, G., Decamps, H., y D`Hausteserre, A. (2001). Bridging human and natural sciences in landscape research. Landscape and Urban Planning, 57, 137-141

Vallina, A. (2017). La evaluación por componentes aplicada al estudio de la calidad visual del paisaje. Comarca de las Fuentes del Narcea, Degaña e Ibias. Ería Revista Cuatrimestral de Geografía, XXXVII (3), 287-302. doi:https://doi.org/10.17811/er.3.2017.287-302

Zube, E,H (1984). "Themes in Landscape Assessment Theory". Landscape Journal, $\mathrm{n}^{\circ} 3$. pp, 104-110. 